\title{
Characterizing the feedback of magnetic field on the differential rotation of solar-like stars
}

\author{
J. Varela ${ }^{a}$, A. Strugarek ${ }^{\mathrm{a}, \mathrm{b}}$, A. S. Brun ${ }^{\mathrm{a}}$ \\ ${ }^{a}$ AIM, CEA/CNRS/University of Paris 7, CEA-Saclay, 91191 Gif-sur-Yvette, France \\ ${ }^{b}$ Département de Physique, Université de Montréal, C.P. 6128 Succ. Centre-Ville, \\ Montréal, QC, H3C-3J7, Canada
}

\begin{abstract}
The aim of this article is to study how the differential rotation of solarlike stars is influenced by rotation rate and mass in presence of magnetic fields generated by a convective dynamo. We use the ASH code to model the convective dynamo of solar-like stars at various rotation rates and masses, hence different effective Rossby numbers. We obtained models with either prograde (solar-like) or retrograde (anti-solar-like) differential rotation. The trends of differential rotation versus stellar rotation rate obtained for simulations including the effect of the magnetic field are weaker compared with hydro simulations $\left(\Delta \Omega \propto\left(\Omega / \Omega_{\odot}\right)^{0.44}\right.$ in the MHD case and $\Delta \Omega \propto\left(\Omega / \Omega_{\odot}\right)^{0.89}$ in the hydro case), hence showing a better agreement with the observations. Analysis of angular momentum transport revealed that the simulations with retrograde and prograde differential rotation have opposite distribution of the viscous, turbulent Reynolds stresses and meridional circulation contributions. The thermal wind balance is achieved in the prograde cases. However, in retrograde cases Reynolds stresses are dominant for high latitudes and near the top of the convective layer. Baroclinic effects are stronger for faster rotating models.
\end{abstract}

Keywords: $50.007 ; 50.008$

Email address: Jacobo.Varela@cea.fr (J. Varela)

Preprint submitted to Journal of $L^{A} T_{E} X$ Templates

June 25, 2018 


\section{Introduction}

It is well known that there is a correlation between magnetic activity and rotation of stars (Durnev, 1976; Noves et al., 1984b; Pizzolato et al., 2003). Rapid rotators show a stronger and more intense magnetic activity (Saar \& Brandenburg, 1999; García et al., 2010) than slower rotators such as the Sun for which the averaged magnetic field is weaker (Pallavicini et al., 1981), therefore a detailed analysis of the differential rotation (DR) is mandatory to understand the magnetic activity of the stars (Donahue et al., 1996).

Dopper imaging (Donati \& Collier Cameron, 1997; Barnes et al., 2005), asteroseismology (Gizon \& Solanki, 2004; Reinhold et al., 2013; García et al., 2014), classical spot models (Lanza et al., 2014) and short-term Fourier-transform (Vida et al., 2014) are methods to infer the differential rotation, while photometric and spectroscopic variability are good indicators of the magnetic activity along the star's activity cycle (Baliunas et al., 1995; Oláh et al., 2009). The combination of both sources of information helps to constrain the trends linking rotation with stellar differential rotation and magnetic activity, data available thanks to recent missions as CoRoT or Kepler. Recent analysis revealed weak dependency between DR and star's rotation $\left(\Delta \Omega \propto \Omega^{0.15}\right)$ Barnes et al., 2005; Reinhold et al., 2013)), larger in case of star's temperature $\left(\Delta \Omega \propto T_{\text {eff }}^{8.92}\right.$ (Barnes et al., 2005; Reinhold et al., 2013) ) and $\Delta \Omega \propto T_{\text {eff }}^{8.6}($ Collier Cameron, 2007)). The differential rotation defined in these communications is $\Delta \Omega=\alpha \Omega_{e q}$ with $\Omega_{e q}$ the angular velocity at the equator and $\alpha$ the relative horizontal shear of the differential rotation between the equator and the pole. $\Omega_{e q}$ and $\alpha$ are deduced from the observations.

Several authors have performed global 3D magnetohydrodynamic (MHD) simulations to model differential rotation and stellar magnetism in the convection zone (Miesch et al., 2006; Ghizaru et al., 2010; Racine et al., 2011; Käpylä et al., 2011, 2014; Augustson et al., 2015; Karak et al., 2015), particularly for solar like stars (Brun et al., 2004; Brown et al., 2010, 2011; Brun et al., 2011). These studies pointed out the large magnetic temporal variability and the critical effect 
of stellar rotation and mass on magnetic field generation through dynamo mechanism, leading in some parameter regimes to configuration with cyclic activity (Gilman, 1983; Gilman \& Miller, 1981; Nelson et al., 2013; Käpvlä et al., 2013; Augustson et al., 2013; Guerrero et al., 2016; Augustson et al., 2015). Several studies pointed out the effect of a stable region underneath the convection zone on the lengthening of the stellar dynamo cycle period (Guerrero et al., 2016; Lawson et al., 2015).

The present study is focused on solar-like stars, G and K stellar classes. Observations indicate that this group of stars show very different magnetic activity (Saar, 1990; Plachinda \& Tarasova, 1999), with short Metcalfe et al. (2010) and long cycles (Baliunas et al., 1995), consequence of the range of masses, rotation, differential rotation, age, effective temperature or metallicity measured (Noves et al., 1984a; Chaplin et al., 2010; Ballard et al., 2014; García et al., 2014; J.-D. do Nascimento et al., 2014). We analyze the correlation between differential rotation and magnetism in solar-like stars using the anelastic spherical harmonic code (ASH) (Brun et al., 2004), performing several convective dynamo MHD simulations for star with different masses and rotation rates (Rossby numbers). One first achievement of the study was to simulate stars with prograde (solar-like) and retrograde (anti-solar-like, equator rotates slower than the poles) differential rotation (Matt et al., 2011; Bessolaz \& Brun, 2011; Gastine et al., 2014; Karak et al., 2015). The aim of this study is to analyze the effect of a magnetic field on the star's differential rotation (Brun, 2004; Fang et al., 2014). We show that the trends of the differential rotation with the stellar mass and rotation for MHD simulations are in better agreement with the observational trends than equivalent hydro simulations (Donahue et al., 1996; Barnes et al., 2005; Reinhold et al., 2013).

The article structure is as follows: section 2; we describe the ASH code, the boundary and initial conditions of the different models as well as the key parameters of each simulation. Section 3; we study the large scale flows for the different models analyzing the time averaged kinetic and magnetic energy of the system, differential rotation as well as the trends of differential rotation versus 
star's rotation rate and mass obtained for hydro and MHD cases. Section 4; we analyze the angular momentum balance in the models studying the mean radial and latitudinal fluxes transport. Section 5; we study the baroclinity and thermal wind balance for typical prograde and retrograde cases. Section 6; conclusion, discussion and perspectives of present study.

\section{Numerical model}

In this section we present the main features of the ASH code, describing the boundary and initial conditions of the numerical model and our choice of the global parameters.

We perform 3D simulations of convective dynamo action that consist in solving the Lantz-Braginski-Roberts (LBR) form of the anelastic MHD equations for a conductive plasma in a rotating sphere (Jones et al., 2011), a formulation that improves the energy conservation in stable stratified regions (Brown et al., 2012; Vasil et al., 2013). The code ASH performs a large-eddy simulation that uses a pseudo-spectral method with the spherical harmonics expansion in the horizontal direction for the entropy $(S)$, magnetic field $(\mathbf{B})$, pressure $(P)$ and mass flux. The density $(\rho)$, entropy, pressure and temperature $(T)$ are linearized about the spherically symmetric background values, denoted by the symbol $\left(^{-}\right)$. The solenoidality of the mass flux and magnetic vector fields is maintained by a streamfunction formalism (Brun et al., 2004). The equations solved by ASH are (Alvan et al., 2014; Augustson et al., 2015):

$$
\begin{gathered}
\boldsymbol{\nabla} \cdot \bar{\rho} \mathbf{v}=0 \\
\bar{\rho} \frac{\partial \mathbf{v}}{\partial t}=-\bar{\rho} \mathbf{v} \cdot \nabla \mathbf{v}-\boldsymbol{\nabla} \bar{\omega}+\frac{S g}{c_{p}} \mathbf{r}+2 \bar{\rho} \mathbf{v} \wedge \mathbf{\Omega}_{0}+\frac{1}{4 \pi}(\nabla \wedge \mathbf{B}) \wedge \mathbf{B}+\nabla \cdot D \\
\bar{\rho} \bar{T} \frac{\partial S}{\partial t}=\bar{\rho} \bar{T} \mathbf{v} \cdot \boldsymbol{\nabla}(\bar{S}+S)-\boldsymbol{\nabla} \cdot \mathbf{q}+\Phi \\
\boldsymbol{\nabla} \cdot \mathbf{B}=0 \\
\frac{\partial \mathbf{B}}{\partial t}=\boldsymbol{\nabla} \wedge[\mathbf{v} \wedge \mathbf{B}-\eta \nabla \wedge \mathbf{B}]
\end{gathered}
$$


with the velocity field $\mathbf{v}=v_{r} \mathbf{r}+v_{\theta} \boldsymbol{\theta}+v_{\varphi} \boldsymbol{\varphi}$, the magnetic field $\mathbf{B}=B_{r} \mathbf{r}+B_{\theta} \boldsymbol{\theta}+$ $B_{\varphi} \boldsymbol{\varphi}$, the angular velocity in the of the rotation frame $\boldsymbol{\Omega}=\Omega_{0} \mathbf{z}, \mathbf{z}$ the direction along the rotation axis, $g$ the magnitude of the gravitational acceleration and $\bar{\omega}=P / \bar{\rho}$ is the reduced pressure in the LBR implementation. The motions not resolved by the numerical mesh are parametrized as effective eddy diffusivities $\nu$, $\kappa$ and $\eta$ that account for the effect of the subgrid-scales transporting momentum, heat and magnetic field. The diffusion tensor $D$ and the dissipative term $\Phi$ are defined as:

$$
\begin{gathered}
D_{i j}=2 \bar{\rho} \nu\left[e_{i j}-\frac{1}{3} \boldsymbol{\nabla} \cdot \mathbf{v} \delta_{i j}\right] \\
\Phi=2 \bar{\rho} \nu\left[e_{i j} e_{i j}-\frac{1}{3}(\boldsymbol{\nabla} \cdot \mathbf{v})^{2}\right]+\frac{4 \pi \eta}{c^{2}} \mathbf{J}^{2}
\end{gathered}
$$

with $e_{i j}$ the stress tensor and $\mathbf{J}=c / 4 \pi \nabla \wedge \mathbf{B}$ the current density. The energy flux $\mathbf{q}$ has a radiation flux and an inhomogeneous turbulent entropy diffusion flux:

$$
\mathbf{q}=\kappa_{r} \bar{\rho} c_{p} \boldsymbol{\nabla}(\bar{T}+T)+\kappa \bar{\rho} \bar{T} \nabla S+\kappa_{0} \bar{\rho} \bar{T} \frac{\partial \bar{S}}{\partial r} \mathbf{r}
$$

with $\kappa_{r}$ the molecular radiation diffusion coefficient, $\kappa_{0}$ the effective thermal diffusivity acting only on the spherically symmetric $(l=0)$ entropy and $c_{p}$ the specific heat at constant pressure. A perfect ideal gas equation is used for the mean state and the fluctuation are linearized:

$$
\begin{gathered}
\bar{P}=(\gamma-1) c_{p} \bar{\rho} \bar{T} / \gamma \\
\rho / \bar{\rho}=P / \bar{P}-T / \bar{T}=P / \gamma \bar{P}-S / c_{p}
\end{gathered}
$$

with $\gamma=5 / 3$ the adiabatic exponent.

The anelastic MHD system of equations requires 12 boundary conditions. Magnetic boundary conditions are perfectly conducting at the lower radial boundary and the magnetic field matches to a potential field in the upper boundary:

$$
\left.B_{r}\right|_{r_{i n}}=\left.\frac{\partial}{\partial r}\left(\frac{B_{\theta}}{r}\right)\right|_{r_{i n}}=\left.\frac{\partial}{\partial r}\left(\frac{B_{\varphi}}{r}\right)\right|_{r_{i n}}=0 \text { and }\left.B_{r}\right|_{r_{\text {out }}}=\nabla \Psi \Rightarrow \Delta \Psi=0
$$

We use an impenetrable and stress free at the top and bottom boundaries. 


$$
v_{r}=\frac{\partial}{\partial r}\left(\frac{v_{\theta}}{r}\right)=\frac{\partial}{\partial r}\left(\frac{v_{\phi}}{r}\right)=0
$$

For the mean state of the entropy $\left.(d \bar{S} / d r)\right|_{r_{\text {out }}}=-3.57 \cdot 10^{-9}$ and $\left.(d \bar{S} / d r)\right|_{r_{\text {in }}}=$ $9.79 \cdot 10^{-4} \mathrm{~cm} \mathrm{~K}^{-1} \mathrm{~s}^{-2}$ for M05 model, $\left.(d \bar{S} / d r)\right|_{r_{\text {out }}}=-9.56 \cdot 10^{-9}$ and $\left.(d \bar{S} / d r)\right|_{r_{\text {in }}}=$ $9.59 \cdot 10^{-3} \mathrm{~cm} \mathrm{~K}^{-1} \mathrm{~s}^{-2}$ for M07 model, $\left.(d \bar{S} / d r)\right|_{r_{\text {out }}}=-3.78 \cdot 10^{-8}$ and $\left.(d \bar{S} / d r)\right|_{r_{\text {in }}}=$ $1.37 \cdot 10^{-2} \mathrm{~cm} \mathrm{~K}^{-1} \mathrm{~s}^{-2}$ for M09 model, $\left.(d \bar{S} / d r)\right|_{r_{\text {out }}}=-3.01 \cdot 10^{-7}$ and $\left.(d \bar{S} / d r)\right|_{r_{\text {in }}}=$ $1.11 \cdot 10^{-2} \mathrm{~cm} \mathrm{~K}^{-1} \mathrm{~s}^{-2}$ for M11 model. Keeping the values of $d \bar{S} /\left.d r\right|_{r_{\text {in }}, r_{\text {out }}}$ fixed at all times in the simulations further implies that the fluctuating $d S / d r$ is set to zero at both bcs.

The simulation is focused in the bulk convection zone, avoiding regions too close the stellar surface. We include the tachocline in the models, defined as the transition between rigid to differential rotation, leading to region with strong shear (Spiegel \& Zahn, 1992). The tachocline plays an important role in the dynamo process of magnetic field generation in solar-like stars as it was reported in simulations performed by several authors (Browning et al., 2006; Racine et al., 2011; Masada \& Sano, 2014; Lawson et al., 2015; Guerrero et al., 2016).

The code uses a realistic background stratification for the profiles of density $(\bar{\rho})$, temperature $(\bar{T}), \nu, \kappa$ and $\eta$. The background stratification is derived from a one-dimensional solar structure model CESAM (Morel, 1997; Brun \& Toomre, 2002). In Fig. 1 we show an example of the gradient of entropy along the star radius (a), and the temporal and azimuthally averaged radial energy fluxes balance as luminosities (b) for the model with 1.1 solar mass and 3 times the rotation rate of the Sun.

The MHD models are initialized from progenitor hydro models in which a small magnetic field perturbation is introduced (several orders smaller than the final magnetic field observed in the simulation). A first analysis of the progenitor hydro models is done in these references Matt et al. (2011); Brun et al. (2015b). The model's resolution is $\left(N_{r}, N_{\theta}, N_{\phi}\right) 769 \times 256 \times 512$ except for model M09 rotating at $\Omega_{*}=3 \Omega_{\odot}$ where $N_{\theta}$ is 512 and $N_{\phi}$ is 1024 . In table 1 we indicate the most relevant parameters of the simulations. The 7 models presented in table 1 


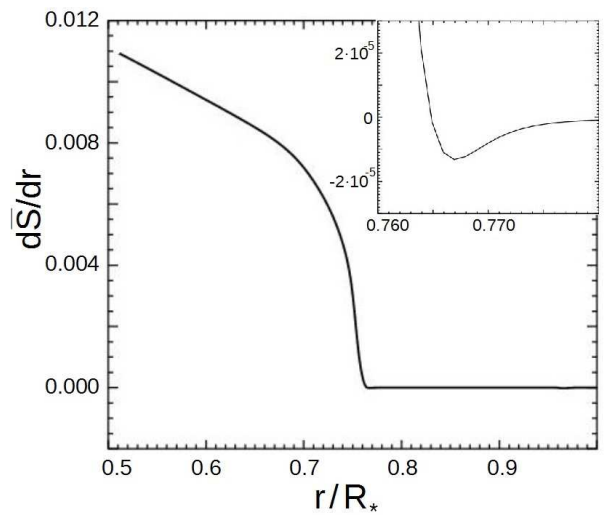

(a)

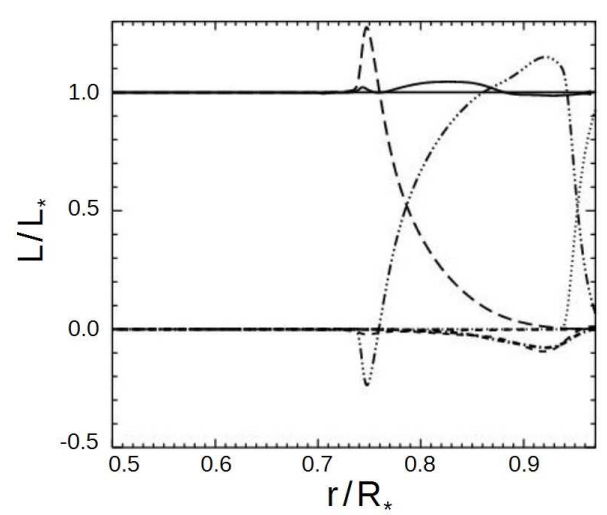

(b)

Figure 1: (a) Entropy gradient along the star radius. We expand the region of the tachocline in the top graph. (b) Time and horizontally averaged radial energy fluxes as luminosities (normalized to the star luminosity). The solid line is the total flux, the long dashed line the radiative flux, the dash-dot-dot-dot line the enthalpy flux, the dotted line the conductive entropy flux, the thick dash-doot line the kinetic energy, the dashed line the viscous diffusion flux and the thick dashed line the Poynting flux.

are named $M A_{x i}$, where $A$ is the mass of the star (in solar masses between 0.5 to 1.1) and $i$ the rotation rate of the star (in solar rotation rate). The subindex indicates slow/anti-solar $(x=s)$ and solar $(x=d)$ differential rotation models (except model $M 11_{d 1}$ also anti-solar). The density scale heights between the top and the base of the convection zone and between the top and the bottom of the model are defined as $N_{\rho_{b c z}}=\ln \left(\rho_{\text {out }} / \rho_{\text {bcz }}\right)$ and $N_{\rho_{\text {tot }}}=\ln \left(\rho_{\text {out }} / \rho_{\text {in }}\right)$. For the $M 05$ model $N_{\rho_{b c z}}=3.25$ and $N_{\rho_{t o t}}=4.70, M 07$ model $N_{\rho_{b c z}}=3.48$ and $N_{\rho_{\text {tot }}}=5.78, M 09$ model $N_{\rho_{b c z}}=3.31$ and $N_{\rho_{\text {tot }}}=5.99, M 11$ model $N_{\rho_{b c z}}=3.28$ and $N_{\rho_{\text {tot }}}=5.60$.

In the present article we omit the discussion of the dynamo characteristics of the models because this will be the topic of a future communication. We focus the analysis on the differential rotation properties for the various parameters 
considered.

\section{Large scale flows and energy content in solar-like stars}

In this section we analyze the differential rotation profiles of the models. The aim of the study is to compare the differential rotation profiles of the hydro and MHD models. We show that the presence of magnetic fields leads to different trends of the differential rotation with stellar rotation rate and mass. Following we analyze the kinetic and magnetic energy contained in the models and how they are distributed between their various components.

\subsection{Large scale flows}

We analyze the differential rotation of the simulations that results from the angular momentum redistribution occurring mostly in the convection zone. The panels of figure 2 show an azimuthal plot of the differential rotation averaged over 10 overturning convective times, defined as $\tau_{c}=\int_{r_{b c r}}^{r_{\text {out }}} d r / \tilde{v}_{r}$ with $\tilde{v}_{r}$ the radial component of the rms velocity in the convection zone, $r_{\text {out }}$ and $r_{b c z}$ the top and the base of the convective layer. We observe that for the simulations $M 07_{s}, M 09_{s}$ and $M 11_{d 1}$ (the figure of the $M 11_{d 1}$ model is not shown) there is an anti-solar differential rotation, with the poles rotating faster than the

equator, while the other cases show a solar-like differential rotation. There is almost no asymmetry in the profiles between the North and South Hemispheres, as expected when the average is performed over an interval long enough with respect to the convective overturning time. We also display radial cuts of the rotation for the MHD cases (red lines) and Hydro progenitor cases (black lines). In cases rotating 1 and 3 times the solar rotation rate, the poles (latitudes of $75^{\circ}$ and higher) are speed up when the magnetic field is present, effect that is stronger as the rotation rate increases. For the $M 05_{d 1}$ and $M 09_{d 1}$ models the poles rotates 18 and $21 \mathrm{nHz}$ faster in the MHD simulation while for the $M 09_{d 3}$ and $M 11_{d 3}$ models the rotation rates increases 155 and $200 \mathrm{nHz}$, pointing out that the effect of the magnetic feedback is larger if the mass of the star increases 
(larger convective velocity hence larger magnetic field and Maxwell stresses, since $M E \sim K E)$. The anti-solar cases show the same behavior, the poles are speed up when the magnetic field is included in the simulation due to the joint action of Maxwell and Reynolds stresses in high latitude regions. If we average the rotation rate between the latitudes $60^{\circ}$ and $75^{\circ}$ we see that for the $M 07_{s}$ and $M 09_{s}$ models the rotation rate is enhanced by 10 and $22 \mathrm{nHz}$. The model that shows a larger contrast between the differential rotation and the star rotation is the case $M 07_{s}$ followed by the models $M 05_{d 1}, M 11_{s}$ and $M 09_{s}$. The gradient of the rotation in the radial direction nearby the tachocline is weaker in all the MHD simulations compared with hydro cases. Anti-solar differential rotation models show an increase of the star's rotation near the tachocline for the MHD cases (recalling that $\Omega$ decreases further out in the convection zone) and a drop for the hydro simulation compared with the solid body rotation of the core, indicating how the presence of the magnetic field modifies the rotation gradients (see Fig. 4 for Maxwell stresses).

Figure 3 shows the trends of the absolute value of the differential rotation and the differential rotation kinetic energy (DRKE) versus the models Rossby number (graphs A and B). The absolute value of the differential rotation drops when Ro increases, undergoing a transition to the anti-solar differential rotation models if $\mathrm{Ro}$ is of the order of 1 or larger with $\Delta \Omega \propto\left(R_{o}\right)^{-0.40 \pm 0.20}$ (we include the standard error of the fit). The DRKE drops too when Ro increases and it is smaller than $10^{6} \mathrm{erg} \cdot \mathrm{cm}^{-3}$ in the anti-solar models because the models are more dominated by non axisymmetric convection (CKE dominant) with $D R K E \propto\left(R_{O}\right)^{-1.31 \pm 0.07}$. The trends of the absolute value of the differential rotation versus stellar rotation (graph $\mathrm{C}$ ), for the regression $\Delta \Omega \propto \Omega^{\alpha}$, is $\Delta \Omega \propto \Omega^{0.44 \pm 0.15}$ in the MHD case and $\Delta \Omega \propto \Omega^{0.89 \pm 0.16}$ in the hydro case. The MHD trend is in better agreement with the observations $\left(\Delta \Omega \propto \Omega^{0.15}\right.$ (Barnes et al., 2005; Reinhold et al., 2013)). The trends of the absolute value of the differential rotation versus stellar mass (graph D) for the regression $\Delta \Omega \propto\left(M / M_{\odot}\right)^{\alpha}$, is $\Delta \Omega \propto\left(M / M_{\odot}\right)^{4.19 \pm 6.86}$ in the MHD case and $\Delta \Omega \propto\left(M / M_{\odot}\right)^{8.68 \pm 3.91}$ in the hydro case. The inclusion of case $M 05_{d 1}$ increase 
significantly the spread of some of the fits and we have chosen to exclude it when necessary to get a better $\chi^{2}$. We are currently investigating the 0.5 solar mass models at various rotation rates to confirm the behavior of very low mass star models. These results will be reported in a future study, including an updated set of model to reduce the gaps in the results, although the current analysis already shows robust trends for the differential rotation. The MHD trend is in better agreement with the observations $\left(\Delta \Omega \propto T_{\text {eff }}^{8.92}\left(\Delta \Omega \propto M^{5.6}\right)\right.$ (Barnes et al., 2005; Reinhold et al., 2013) or $\Delta \Omega \propto T_{\text {eff }}^{8.6}\left(\Delta \Omega \propto M^{5.4}\right.$ ) (Collier Cameron, 2007)), confirming that more massive stars have larger differential rotation and predicting more accurately the observed trends.

The MHD simulations show how the impact of the magnetic field changes the angular momentum redistribution tending to make the DR more rigid and less sensitive to global parameter changes. In the next section we will perform a detailed analysis of this balance for all the models.

\subsection{Energetic content}

Table 2 indicates the kinetic and magnetic energy of the models averaged in time over the domain (mostly convective zone). Most of the system energy is in form of kinetic energy (KE) for the models $M 05_{d 1}, M 07_{s}$ and $M 09_{s}$ and the magnetic energy (ME) is at least a $3 \%$ of the total energy in the models $M 09_{d 1}, M 09_{d 3}, M 11_{d 1}$ and $M 11_{d 3}$. Between 1 to $15 \%$ of the star's luminosity is required to maintain $\Omega(r, \theta)$ and the conversion to mean toroidal component of magnetic energy (TME) depends of the Elsasser number of each model. A detailed analysis of the energy transfer including physical explanations is performed in Brun et al. (2011, 2015a). The differential rotation kinetic energy component (DRKE) is dominant in the models $M 09_{d 3}, M 11_{d 3}$ and $M 05_{d 1}$, while for the models $M 07_{s}, M 09_{s}$ and $M 11_{d 1}$ it is the turbulent convective kinetic energy (CKE). Only in the model $M 09_{d 1}$ there is almost the same amount of energy in both components. The meridional circulation kinetic energy (MCKE) is negligible in all cases except for the $M 09_{s}$ model where it is a $12 \%$ of the total KE. The mean toroidal component of magnetic energy (TME) is dominant 
in all simulations, followed by the fluctuation of magnetic energy (FME) that accounts for $20 \%$ of the magnetic energy (value than reaches $30 \%$ in $M 11_{d 1}$ model). The mean poloidal component of the magnetic energy (PME) reaches only $2 \%$ of the total energy, except in the anti-solar models $M 09_{s}(23 \%)$ and $M 11_{d 1}(7 \%)$. The models with larger differential rotation at the top of the convection zone (calculated as $\Delta \Omega=\Omega\left(\theta=0^{\circ}\right)-\Omega\left(\theta=60^{\circ}\right)$ ) are $M 09_{d 3}, M 11_{d 1}$ and $M 11_{d 3}$. The largest ratio between the differential rotation and the stellar rotation is observed for the anti-solar and low mass models. Only the models $M 07_{s}$ and $M 09_{s}$ show a larger ratio in the MHD models compared with the hydro simulations.

\section{Angular momentum balance}

Since we wish to focus this paper on stellar DR we now assess which mechanisms maintain it. Hence in this section we study the main physical processes that redistribute the angular momentum in the convective layer. We show the angular momentum balance of the models $M 09_{s}$ and $M 09_{d 3}$ as an example of anti-solar and solar differential rotation cases (see Fig 4). The angular momentum transport can be described by the mean radial $F_{r}$ and latitudinal $F_{\theta}$

angular momentum fluxes (Elliott et al., 2000; Brun et al., 2004). We integrate these values in colatitude and in radius, to calculate the net fluxes through respectively cones of various angles and concentric spheres of various radii:

$$
\begin{aligned}
& \mathcal{F}_{r}(r)=\int_{0}^{\pi} F_{r}(r, \theta) r^{2} \sin \theta d \theta \\
& \mathcal{F}_{\theta}(\theta)=\int_{r_{\text {in }}}^{r_{\text {out }}} F_{\theta}(r, \theta) r \sin \theta d r
\end{aligned}
$$

decomposing this transport in viscous diffusion, turbulent Reynolds stresses, meridional circulation (axisymmetric Reynolds stresses from differential rotation + Coriolis), axisymmetric and turbulent Maxwell stresses contributions. Figure 4 shows the $\mathcal{F}_{r}$ and $\mathcal{F}_{\theta}$ integrated angular momentum fluxes for the models $M 09_{s}$ and $M 09_{d 3}$. 
The solid line indicates the total net momentum fluxes, almost null for $\mathcal{F}_{r}(r)$ and small for $\mathcal{F}_{\theta}(\theta)$, pointing out that the simulation is in a robust statiscally stationary state. The viscous diffusion term for $\mathcal{F}_{r}(r)$ angular momentum flux is positive in the anti-solar case but negative in the solar case, indicating that in the anti-solar case there is a radially outward transport of angular momentum while in the solar case the transport is inward, consequence of the different radial gradient of the rotation in the models. In the anti-solar case the outward transport is enhanced by the meridional circulation and compensated by the turbulent Reynolds stresses, opposite to what is realized in the solar case. The role of the Maxwell stresses is larger in the solar case than in the antisolar model, showing a larger peak of the axisymmetric component near the tachocline because the $\mathrm{ME}$ in $M 09_{d 3}$ model is one order larger than in the $M 09_{s}$ case), compensating all the other components. For both models there is a small transfer of angular momentum in the stable radiative region nearby the tachocline.

The $\mathcal{F}_{\theta}(\theta)$ angular momentum flux balance shows a more complicated decomposition between components than $\mathcal{F}_{r}(r)$. In the anti-solar case the dominant terms are the turbulent Reynolds stresses and the meridional circulation, which balance each other, with a smaller input of the viscosity (positive near the equator and negative at high latitudes) and the axisymmetric Maxwell stress (enhancing the turbulent Reynolds stresses near the equator). In the solar case the dominant component is the meridional circulation, enhanced near the equator and at high latitudes by the turbulent Reynolds stresses, and compensated by the viscous diffusion and the axisymmetric and turbulent Maxwell stresses (Fang et al., 2014). These results point out that in the solar case the role of the Maxwell stresses is important for the angular momentum balance, leading to a configuration with modulated activity, while in this anti-solar case the role of the magnetic fields is smaller and the modulation of the activity weaker, but large enough to increase the differential rotation of the models. If Maxwell stresses are large enough the star's differential rotation decreases due to the quenching effect of the magnetic fields. The decrease of the differential rotation leads to a 
weaker magnetic field but the Reynolds stresses are opposed to the drop of the differential rotation. The interplay between Maxwell and Reynolds stresses can drive a non stationary evolution of the differential rotation and the magnetic fields of the star that can show chaotic or regular variabilities (see for instance Brun et al. (2005); Brown et al. (2011)).

To summarize, we show in Figure 5 the leading components of the angular momentum balance in all the models as an histogram (except $M 11_{1 d}$ model), where the $\mathcal{F}_{r}(r)$ angular momentum flux is further integrated in radius and $\mathcal{F}_{\theta}(\theta)$ angular momentum flux in latitude (only over the North Hemisphere). The anti-solar cases share same patterns; integrated $\mathcal{F}_{r}(r)$ shows the balance between viscous diffusion and meridional circulation with the turbulent Reynolds stresses, the opposite scenario than in the solar-like differential rotation models. For the integrated $\mathcal{F}_{\theta}(\theta)$ the turbulent Reynolds stresses and the viscous diffusion are balanced by the meridional circulation, while in the solar-like differential rotation models the balance is more complex, especially for the cases with $3 \Omega$ where the Maxwell axisymmetric stresses have an important role. For a larger stellar rotation, the ratio between $\mathrm{ME}$ and $\mathrm{KE}$ increases leading to a stronger feedback between the fields and the flows. This is easily understood by Table 1 given the larger value of the Elsasser number for the fastly rotating cases which modifies the scaling of the magnetic fields amplitude (Christensen, 2010; Brun et al., 2015a).

Figure 6 shows the temporal and longitudinal average of the meridional circulation for the models $M 09_{s}$ and $M 09_{d 3}$. The meridional circulation is represented by the isocontours of the stream function $\psi$ defined as (Miesch et al., 2000):

$$
\begin{aligned}
\left\langle\bar{\rho} v_{r}\right\rangle & =\frac{1}{r^{2} \sin \theta} \frac{\partial \psi}{\partial \theta} \\
\left\langle\bar{\rho} v_{\theta}\right\rangle & =-\frac{1}{r \sin \theta} \frac{\partial \psi}{\partial r}
\end{aligned}
$$

The meridional circulation is driven by the interplay of the buoyancy and Coriolis forces, pressure gradients, viscosity, Reynolds and Maxwell tensors acting 
on the zonal flows, leading to deviations in the magnetostrophic equilibrium, consequence of the fluctuating essence of the convection. The system reaction to restore the equilibrium yields a redistribution of the angular momentum by a mechanism called gyroscopic pumping (Miesch et al., 2006; McIntvre, 2007; Brun et al., 2011), driving the meridional circulation. In figure 6, the cells with red color indicate counterclockwise rotation and the blue color clockwise rotation. $M 09_{s}$ model shows an unicellular meridional circulation, counterclockwise rotating at the North Hemisphere and clockwise at the South Hemisphere. $M 09_{d 3}$ model shows a multicellular meridional circulation, consequence of a stronger alignment of the cells with the rotation axis as the model rotation rate increases. The Hydro version of $M 09_{s}$ and $M 09_{d 3}$ models shows similar meridional circulation. For the other MHD models, same behavior is observed: antisolar (retrograde) cases show unicellular flow and prograde ones multi-cellular, increasing the number of cells with the rotation rate (Featherstone \& Miesch, 2015).

Figure 7 shows an example of time-latitude diagrams of the $\varphi$ component of the magnetic field $\left(B_{\varphi}\right)$, the torsional oscillations (defined as the difference between the average rotation and the instantaneous rotation of the star in Covas et al. (2000); Spruit (2003); Covas et al. (2005); Brun \& Rempel (2009)), the temperature fluctuations (defined as the difference between the average temperature and the instantaneous temperature) as well as the turbulent Reynolds stresses and Maxwell stresses components of the latitudinal angular momentum flux at the tachocline $\left(r / r_{*}=0.54\right.$, left panels $)$ and at the top of the convective layer $\left(r=r_{\text {out }}\right.$, right panels) for the model $M 05_{d 1}$. There is a polarity inversion of $B_{\varphi}$ nearby the tachocline between the day 3000 and 4000 of simulated time, while there are successive inversions with much shorter period at the top of the convective layer. The evolution of the $B_{\varphi}$ nearby the tachocline is correlated with the torsional and temperature oscillations at the top of the convection zone, showing that the star's rotation is decelerated and the temperature drops near the equator when $B_{\varphi}$ increases after the polarity inversion. The evolution of the turbulent Reynolds stresses component of the latitudinal 
angular momentum flux show hints of correlation with the fast-oscillating $B_{\varphi}$ at the top of the convection zone. A longer time-series would though be needed to further confirm this correlation is significant compared to epochs where the oscillating $B_{\varphi}$ is weaker. At the tachocline, the turbulent Reynolds stresses show stable patterns nearby the equator, isolated among hemispheres. The Maxwell stresses components of the latitudinal angular momentum flux at the tachocline follow $B_{\varphi}$ evolution, showing a minimum during the polarity inversion. Near the upper boundary, Maxwell stresses increase with $B_{\varphi}$, indicating a potentially significant feedback effect over the star's differential rotation.

\section{Baroclinity and Thermal Wind balance}

The effect of rotation upon convection leads to latitudinal heat transport that establishes gradients in temperature and entropy. To analyze this effect, Figure 8 shows azimuthal plots of temperature and entropy averaged over 10 overturning convective times for the models $M 09_{s}$ and $M 09_{d 3}$.

There are gradients of entropy and temperature in the convective layer, particularly large in latitude when comparing values at the equator and poles although gradients in radius are large too at high latitudes. The rotation is slower in $M 09_{s}$ model reason why the gradients are small compared with $M 09_{d 3}$ model (because $\frac{\partial\langle S\rangle}{\partial \theta}=\frac{2 \Omega_{0} r c_{p}}{g} \frac{\partial\left\langle v_{\phi}\right\rangle}{\partial z}$, see Brown et al. (2008)). Hydro simulations show stronger gradients than the MHD cases in agreement with the overall large angular velocity contrast. Figure 9 shows the temperature and entropy constrast (defined between the latitude $60^{\circ}$ and the equator) with the star's rotation. The trends for the temperature contrast, defined as $\Delta T \propto\left(\Omega / \Omega_{\odot}\right)^{\alpha}$, is $\Delta T \propto\left(\Omega / \Omega_{\odot}\right)^{0.59 \pm 0.25}$ in the MHD case and $\Delta T \propto\left(\Omega / \Omega_{\odot}\right)^{0.66 \pm 0.26}$ in the hydro case. The trends for the entropy contrast (weighted by the star's luminosity) is $\Delta S\left(L_{\odot} / L\right) \propto\left(\Omega / \Omega_{\odot}\right)^{0.81 \pm 0.52}$ in the MHD case and $\Delta S\left(L_{\odot} / L\right) \propto\left(\Omega / \Omega_{\odot}\right)^{0.83 \pm 0.81}$ in the hydro case. So we see that the temperature and entropy contrasts increase with the star's rotation in agreement with thermal wind-like balance, but with a weaker trend in the MHD 
cases.

The presence of gradients in temperature and entropy leads to a mismatch of the iso-surfaces of mean density and pressure that is named baroclinicity and appears in the vorticity equation (Zahn, 1992; Miesch et al., 2006; Brun et al., 2010). The baroclinic term contributes to breaking Taylor constraint of a cylindrical mean flow yielding more complex (conical) angular velocity profiles (Kitchatinov \& Ruediger, 1995). In figures 10 and 11 we show for the models $M 09_{s}$ and $M 09_{d 3}$ if the departure from cylindricity of the differential rotation is accounted for mainly by the baroclinic term or if we must consider other effects. The balance of the mean zonal components of the curl of the momentum (time and azimuthal averaged defined with the symbol \langle\rangle ) can be expressed as (Elliott et al., 2000; Brun et al., 2004; Strugarek et al., 2011):

$$
\begin{gathered}
2 \Omega_{0} \frac{\partial v_{\phi}}{\partial z}=-\left\langle(\boldsymbol{\omega} \cdot \nabla) v_{\phi}+\frac{\omega_{\phi} v_{r}}{r}+\frac{\omega_{\phi} v_{\theta} \cot \theta}{r}\right\rangle+\left\langle(\mathbf{v} \cdot \nabla) \omega_{\phi}+\frac{v_{\phi} \omega_{r}}{r}+\frac{v_{\phi} \omega_{\theta} \cot \theta}{r}\right\rangle \\
-\left\langle\omega_{\phi} v_{r}\right\rangle \frac{d l n \bar{\rho}}{d r}+\frac{g}{r c_{p}} \frac{\partial\langle S\rangle}{\partial \theta}+\frac{1}{r \bar{\rho} c_{p}} \frac{d \bar{S}}{d r} \frac{\partial\langle P\rangle}{\partial \theta}+\frac{1}{r}\left[\frac{\partial}{\partial r}\left(r\left\langle A_{\theta}\right\rangle\right)-\frac{\partial}{\partial \theta}\left\langle A_{r}\right\rangle\right] \\
+\left\langle-\frac{1}{c \bar{\rho}}(\mathbf{B} \cdot \nabla) j_{\phi}-\frac{j_{r} B_{\phi}}{c \bar{\rho} r}-\frac{j_{\theta} B_{\phi} \cot \theta}{c \bar{\rho} r}\right\rangle+\left\langle\frac{1}{c \bar{\rho}}(\mathbf{j} \cdot \nabla) B_{\phi}+\frac{j_{\phi} B_{r}}{c \bar{\rho} r}+\frac{j_{\phi} B_{\theta} \cot \theta}{c \bar{\rho} r}\right\rangle \\
+\left(\frac{\left\langle B_{r} j_{\phi}\right\rangle}{c \bar{\rho}}-\frac{\left\langle B_{\phi} j_{r}\right\rangle}{c \bar{\rho}}\right) \frac{d \ln \bar{\rho}}{d r}
\end{gathered}
$$

where:

$$
\begin{gathered}
\frac{\partial}{\partial z}=\cos \theta \frac{\partial}{\partial r}-\sin \theta \frac{\partial}{\partial \theta} \\
A_{r}=\frac{1}{\bar{\rho}}\left[\frac{1}{r^{2}} \frac{\partial\left(r^{2} D_{r r}\right)}{\partial r}+\frac{1}{r \sin \theta} \frac{\sin \theta D_{\theta r}}{\partial \theta}-\frac{D_{\theta \theta}+D_{\phi \phi}}{r}\right] \\
A_{\theta}=\frac{1}{\bar{\rho}}\left[\frac{1}{r^{2}} \frac{\partial\left(r^{2} D_{r \theta}\right)}{\partial r}+\frac{1}{r \sin \theta} \frac{\sin \theta D_{\theta \theta}}{\partial \theta}+\frac{D_{\theta r}-\cot \theta D_{\phi \phi}}{r}\right]
\end{gathered}
$$

with the definitions: $\boldsymbol{\omega}=\boldsymbol{\nabla} \times \mathbf{v}$ the vorticity, $\mathbf{j}$ the current density, $c_{p}$ the specific heat at constant pressure and $D_{i j}$ the viscous stress tensor. The first term in the equation is the stretching by velocity gradients, the second term the advection by the flow, the third term the compressibility, the fourth and fifth terms the baroclinic terms due to non-alignment of density and pressure gradients and of 
the departures from the adiabatic stratification, the term number 6 the diffusion by viscous stresses and the last 3 terms are the magnetic contributions to the "shear" and "transport" of the magnetic field by the current and compressibility.

We expect that the thermal wind is stronger as the star's rotation increases according to the increase of temperature and entropy gradients. For the antisolar case the baroclinic term is the leading component in all the domain except near the upper boundary at high latitudes, where the viscous and Reynolds stresses (particularly the advection) are in opposition to the baroclinic term. For the solar case the baroclinic term is more dominant than in the anti-solar model. Only very close to the top of the convective layer there are meaningful signatures of the viscous stresses and the stretching component of the Reynolds stresses, in opposition to the baroclinic term near the equator, as well as the advection component of the Reynolds stresses at mid and high latitudes. The magnetic contributions are mainly negligible with only weak signatures near the tachocline. In summary, for the anti-solar model the Reynolds stresses and viscous effects should be considered especially at high latitudes, while for the solar case the baroclinic term dominates in the entire domain except very close to the surface.

To summarize, we calculated the percentage of departure from cylindricity of the differential rotation by the baroclinic term, defined as $|L H S-B A R / L H S|$ where LHS and BAR (baroclinic term) are integrated in radius and latitude. Following thermal wind balance we expect the baroclinic term to increase with rotation (as Fig. 9,10 and 11 confirm), but the baroclinic term becomes relatively less important in the overall balance $\left(\Delta S \propto \Omega^{n}\right.$ with $\left.0<n<1\right)$ and the difference is mostly compensated by Reynolds stresses. The trend obtained is $|L H S-B A R / L H S| \approx \Omega^{0.26 \pm 0.48}$ in the MHD cases.

\section{Conclusions and dicussion}

The distribution of the energy reservoir in solar-like stars for anti-solar and solar differential rotation cases is different. The main part of the energy in both 
cases is KE but in the models with solar differential rotation there is a non negligible proportion of ME. We expect this ratio to increase even further as star's rotation increases beyond 3 times the rotation of the Sun reaching equipartition, even super equipartition (as in Augustson et al. (2016); Brun et al. (2015a)) . The KE in solar differential rotation cases is mainly DRKE (more dominant if the star rotation rate is larger) while in the anti-solar cases it is CKE, pointing out that the convective dynamo in the anti-solar cases is dominated by convective motions, a characteristic of $\alpha^{2}$ dynamos, but in the solar cases the $\Omega$ effect can be large enough to build up $\alpha-\Omega$ or $\alpha^{2}-\Omega$ dynamos. A detailed analysis of the convective dynamos in each model of this article will be carried out in a future publication.

There are essential differences in the rotation profiles comparing simulations with and without magnetic fields. The presence of magnetic fields drives a speed up of the poles and a slowdown of the equator compared with the hydro simulations. The speed up effect is stronger as the star's rotation rate increases, because the enhancement of the star magnetic field leads to a larger transfer of angular momentum from the equator to the poles. The reason of the poles speed up in the anti-solar cases is due to magnetic field opposing Reynolds stresses.

The trends between the star's differential rotation, rotation rate and mass shows a better agreement with observations for the MHD cases than for the hydro simulations. There is a weaker dependency of the differential rotation with the star rotation rate (there is a drop of the power factor from 0.89 in the hydro cases to 0.44 in the MHD simulations) and star's mass (from 8.68 in the hydro cases to 4.19 in the MHD simulations), as well as flatter profiles if we compare the differential rotation and DRKE with the model Ro. This could explain recent observations of solar-like stars since they are seen to possess magnetic fields. To fully account the effect of the magnetic field in the stars differential rotation using models with different masses and rotation rates, a systematic analysis of the models parameters is required. In future communications we will refine the trends obtained in the present communication adding new models to the analysis, but the current analysis already shows robust trends. 
The radial angular momentum flux balance in the anti-solar differential rotation case show opposite characteristics than the solar one; there is a radially outward transport of angular momentum by Reynolds stresses in the anti-solar case while it is inward in the solar case. The latitudinal angular momentum flux balance in the anti-solar case is dominated by the opposition between convective and meridional circulation motions, but for the solar case there is a more complex interplay between components dominated by the meridional circulation and compensated by viscous and magnetic stresses.

The baroclinic term in the anti-solar differential rotation case is not the dominant component at high latitudes near the top of the convective layer, the effect of the Reynolds stresses and viscosity are important and should be considered to explain the resulting differential rotation of the model. In the solar case, particularly for the model with the largest rotation rate, the baroclinic term is dominant and the other components are almost negligible in the entire domain with only small contributions very close to the top of the convective layer. Baroclinic effects are stronger as star's rotation increases.

\section{Acknowledgements}

We have received funding by the European Commission's Seventh Framework Programme under the grant agreement SPACEINN (project number 312844) and ERC STARS2 (207430). We thank S. Matt and O. Do Cao for performing most of the HD models. A. Strugarek is a National Postdoctoral Fellow at the Canadian Institute of Theoretical Astrophysics, and acknowledges support from the Canadas Natural Sciences and Engineering Research Council. We extend our thanks to CNES for PLATO science support and INSU/PNST for our grant. We thank the support of Genci center under grant 1623 and Prace allocation ra1964. 


\section{References}

\section{References}

Alvan, L., Brun, A. S., \& Mathis, S. (2014). Theoretical seismology in 3D: nonlinear simulations of internal gravity waves in solar-like stars. $A \& A, 565$, 1-26. doi:10.1051/0004-6361/201323253, arXiv:1403.4052

Augustson, K., Brun, A. S., Miesch, M., \& Toomre, J. (2015). Grand Minima and Equatorward Propagation in a Cycling Stellar Convective Dynamo. ApJ, 809, 1-25. doi:10.1088/0004-637X/809/2/149.

Augustson, K. C., Brun, A. S., \& Toomre, J. (2013). Dynamo Action and Magnetic Cycles in F-type Stars. ApJ, r7\%, 1-22. doi:10.1088/0004-637X/777/2/153

Augustson, K. C., Brun, A. S., \& Toomre, J. (2016). The Magnetic Furnace: Intense Core Dynamos in B-stars. ArXiv e-prints, . arXiv:1603.03659

Baliunas, S. L., Donahue, R. A., Soon, W. H., Horne, J. H., Frazer, J., WoodardEklund, L., Bradford, M., Rao, L. M., Wilson, O. C., Zhang, Q., Bennett, W., Briggs, J., Carroll, S. M., Duncan, D. K., Figueroa, D., Lanning, H. H., Misch, T., Mueller, J., Noyes, R. W., Poppe, D., Porter, A. C., Robinson, C. R., Russell, J., Shelton, J. C., Soyumer, T., Vaughan, A. H., \& Whitney, J. H. (1995). Chromospheric variations in main-sequence stars. ApJ, 438, 269-287. doi:10.1086/175072.

Ballard, S., Chaplin, W. J., Charbonneau, D., Désert, J.-M., Fressin, F., Zeng, L., Werner, M. W., Davies, G. R., Silva Aguirre, V., Basu, S., Christensen-Dalsgaard, J., Metcalfe, T. S., Stello, D., Bedding, T. R., Campante, T. L., Handberg, R., Karoff, C., Elsworth, Y., Gilliland, R. L., Hekker, S., Huber, D., Kawaler, S. D., Kjeldsen, H., Lund, M. N., \& Lundkvist, M. (2014). Kepler-93b: A Terrestrial World Measured to within $120 \mathrm{~km}$, and a Test Case for a New Spitzer Observing Mode. ApJ, 790, 1-16. doi: $10.1088 / 0004-637 X / 790 / 1 / 12$ 
Barnes, J. R., Collier Cameron, A., Donati, J.-F., James, D. J., Marsden, S. C., \& Petit, P. (2005). The Dependence of Differential Rotation on Temperature and Rotation. MNRAS, 357, L1-L5. doi $10.1111 / j .1745-3933.2005 .08587 . x$

Bessolaz, N., \& Brun, A. S. (2011). Towards a 3D dynamo model of the PMS star BP Tau. AN, 332, 1045-1051. doi:10.1002/asna.201111612.

Brown, B. P., Browning, M. K., Brun, A. S., Miesch, M. S., \& Toomre, J. (2008). Rapidly Rotating Suns and Active Nests of Convection. ApJ, 689, 1354-1372. doi $10.1086 / 592397$.

Brown, B. P., Browning, M. K., Brun, A. S., Miesch, M. S., \& Toomre, J. (2010). Persistent Magnetic Wreaths in a Rapidly Rotating Sun. ApJ, 711, 424-438. doi $10.1088 / 0004-637 \mathrm{X} / 711 / 1 / 424$

Brown, B. P., Miesch, M. S., Browning, M. K., Brun, A. S., \& Toomre, J. (2011). Magnetic Cycles in a Convective Dynamo Simulation of a Young Solar-type Star. ApJ, 731, 1-19. doi $10.1088 / 0004-637 \mathrm{X} / 731 / 1 / 69$.

Brown, B. P., Vasil, G. M., \& Zweibel, E. G. (2012). Energy Conservation and Gravity Waves in Sound-proof Treatments of Stellar Interiors. Part I. Anelastic Approximations. ApJ, 756, 1-20. doi $10.1088 / 0004-637 \mathrm{X} / 756 / 2 / 109$ arXiv:1207.2804

Browning, M. K., Miesch, M. S., Brun, A. S., \& Toomre, J. (2006). Dynamo Action in the Solar Convection Zone and Tachocline: Pumping and Organization of Toroidal Fields. ApJ, 648, L157-L160. doi $10.1086 / 507869$

Brun, A. S. (2004). On the interaction between differential rotation and magnetic fields in the Sun. Sol. Phys., 220, 333-345. doi $10.1023 /$ B: SOLA.0000031384.75850.68.

Brun, A. S., Antia, H. M., \& Chitre, S. M. (2010). Is the solar convection zone in strict thermal wind balance? A\&A, 510, 1-7. doi $10.1051 / 0004-6361 / 200913166$. 
Brun, A. S., Browning, M. K., Dikpati, M., Hotta, H., \& Strugarek, A. (2015a).

Recent Advances on Solar Global Magnetism and Variability. Space Sci. Rev., 196, 101-136. doi $10.1007 / \mathrm{s} 11214-013-0028-0$.

Brun, A. S., Browning, M. K., \& Toomre, J. (2005). Simulations of Core Convection in Rotating A-Type Stars: Magnetic Dynamo Action. ApJ, 629, 461-481. doi:10.1086/430430

Brun, A. S., García, R. A., Houdek, G., Nandy, D., \& Pinsonneault, M. (2015b). The Solar-Stellar Connection. Space Sci. Rev., 196, 303-356. doi $10.1007 / \mathrm{s} 11214-015-0157-8$.

Brun, A. S., Miesch, M. S., \& Toomre, J. (2004). Global-Scale Turbulent Convection and Magnetic Dynamo Action in the Solar Envelope. ApJ, 614, 1073-1098. doi $10.1086 / 423835$.

Brun, A. S., Miesch, M. S., \& Toomre, J. (2011). Modeling the Dynamical Coupling of Solar Convection with the Radiative Interior. ApJ, 742, 1-20. doi $10.1088 / 0004-637 \mathrm{X} / 742 / 2 / 79$

Brun, A. S., \& Rempel, M. (2009). Large Scale Flows in the Solar Convection Zone. Space Sci. Rev., 144, 151-173. doi 10.1007/s11214-008-9454-9.

Brun, A. S., \& Toomre, J. (2002). Turbulent Convection under the Influence of Rotation: Sustaining a Strong Differential Rotation. ApJ, 570, 865-885. doi $10.1086 / 339228$, arXiv: astro-ph/0206196

Chaplin, W. J., Appourchaux, T., Elsworth, Y., García, R. A., Houdek, G., Karoff, C., Metcalfe, T. S., Molenda-Żakowicz, J., Monteiro, M. J. P. F. G., Thompson, M. J., Brown, T. M., Christensen-Dalsgaard, J., Gilliland, R. L., Kjeldsen, H., Borucki, W. J., Koch, D., Jenkins, J. M., Ballot, J., Basu, S., Bazot, M., Bedding, T. R., Benomar, O., Bonanno, A., Brandão, I. M., Bruntt, H., Campante, T. L., Creevey, O. L., Di Mauro, M. P., Doğan, G., Dreizler, S., Eggenberger, P., Esch, L., Fletcher, S. T., Frandsen, S., Gai, N., Gaulme, P., Handberg, R., Hekker, S., Howe, R., Huber, D., Korzennik, 
S. G., Lebrun, J. C., Leccia, S., Martic, M., Mathur, S., Mosser, B., New, R., Quirion, P.-O., Régulo, C., Roxburgh, I. W., Salabert, D., Schou, J., Sousa, S. G., Stello, D., Verner, G. A., Arentoft, T., Barban, C., Belkacem, K., Benatti, S., Biazzo, K., Boumier, P., Bradley, P. A., Broomhall, A.-M., Buzasi, D. L., Claudi, R. U., Cunha, M. S., D'Antona, F., Deheuvels, S., Derekas, A., García Hernández, A., Giampapa, M. S., Goupil, M. J., Gruberbauer, M., Guzik, J. A., Hale, S. J., Ireland, M. J., Kiss, L. L., Kitiashvili, I. N., Kolenberg, K., Korhonen, H., Kosovichev, A. G., Kupka, F., Lebreton, Y., Leroy, B., Ludwig, H.-G., Mathis, S., Michel, E., Miglio, A., Montalbán, J., Moya, A., Noels, A., Noyes, R. W., Pallé, P. L., Piau, L., Preston, H. L., Roca Cortés, T., Roth, M., Sato, K. H., Schmitt, J. et al. (2010). The asteroseismic potential of Kepler: first results for solar-type stars. ApJ, 713, L169-L175. doi $10.1088 / 2041-8205 / 713 / 2 /$ L169.

Christensen, U. R. (2010). Dynamo Scaling Laws and Applications to the Planets. Space Sci. Rev., 152, 565-590. doi:10.1007/s11214-009-9553-2.

Collier Cameron, A. (2007). Differential rotation on rapidly rotating stars. AN, 328, 1030-1033. doi 10.1002/asna.200710880

Covas, E., Moss, D., \& Tavakol, R. (2005). Dynamo models and differential rotation in late-type rapidly rotating stars. A\&A, 429, 657-665. doi $10.1051 / 0004-6361: 20041741$.

Covas, E., Tavakol, R., Moss, D., \& Tworkowski, A. (2000). Torsional oscillations in the solar convection zone. A\&A, 360, L21-L24.

Donahue, R. A., Saar, S. H., \& Baliunas, S. L. (1996). A Relationship between Mean Rotation Period in Lower Main-Sequence Stars and Its Observed Range. ApJ , 466, 384-391. doi:10.1086/177517.

Donati, J.-F., \& Collier Cameron, A. (1997). Differential rotation and magnetic polarity patterns on $\mathrm{AB}$ Doradus. MNRAS, 291, 1-19. doi $10.1093 / \mathrm{mnras} / 291.1 .1$. 
Durney, D. R. (1976). On the constancy along cylinders of the angular velocity in the solar convection zone. ApJ, 204, 589-596. doi:10.1086/154207.

Elliott, J. R., Miesch, M. S., \& Toomre, J. (2000). Turbulent Solar Convection and Its Coupling with Rotation: The Effect of Prandtl Number and Thermal Boundary Conditions on the Resulting Differential Rotation. ApJ, 533, 546556. doi:10.1086/308643.

Fang, F., Fan, Y., \& McIntosh, S. W. (2014). Rotating Solar Jets in Simulations of Flux Emergence with Thermal Conduction. ApJ, 789, 1-6. doi $10.1088 / 2041-8205 / 789 / 1 /$ L19.

Featherstone, N. A., \& Miesch, M. S. (2015). Meridional Circulation in Solar and Stellar Convection Zones. ApJ, 804, 1-23. doi:10.1088/0004-637X/804/1/67, arXiv: 1501.06501

García, R. A., Ceillier, T., Salabert, D., Mathur, S., van Saders, J. L., Pinsonneault, M., Ballot, J., Beck, P. G., Bloemen, S., Campante, T. L., Davies, G. R., do Nascimento, J.-D., Jr., Mathis, S., Metcalfe, T. S., Nielsen, M. B., Suárez, J. C., Chaplin, W. J., Jiménez, A., \& Karoff, C. (2014). Rotation and magnetism of Kepler pulsating solar-like stars Towards asteroseismically calibrated age-rotation relations. A\&A, 572, 1-15. doi $10.1051 / 0004-6361 / 201423888$.

García, R. A., Mathur, S., Salabert, D., Ballot, J., Régulo, C., Metcalfe, T. S., \& Baglin, A. (2010). CoRoT Reveals a Magnetic Activity Cycle in a Sun-Like Star. Science, 329, 1032-1032. doi 10.1126/science.1191064.

Gastine, T., Yadav, R. K., Morin, J., Reiners, A., \& Wicht, J. (2014). From solar-like to anti-solar differential rotation in cool stars. MNRAS, 438, L76L80. doi $10.1093 / \mathrm{mnrasl} / \mathrm{slt} 162$

Ghizaru, M., Charbonneau, P., \& Smolarkiewicz, P. K. (2010). Magnetic cycles in global large-eddy simulations of solar convection. ApJ, 715, L133-L137. doi $10.1088 / 2041-8205 / 715 / 2 /$ L133. 
Gilman, P. A. (1983). Dynamically consistent nonlinear dynamos driven by convection in a rotating spherical shell. II - Dynamos with cycles and strong feedbacks. ApJS, 53, 243-268. doi 10.1086/190891.

Gilman, P. A., \& Miller, J. (1981). Dynamically consistent nonlinear dynamos driven by convection in a rotating spherical shell. ApJS, (pp. 211-238). doi: $10.1086 / 190743$.

Gizon, L., \& Solanki, S. K. (2004). Measuring Stellar Differential rotation with asteroseismology. Sol. Phys., 220, 169-184. doi $10.1023 /$ B: SOLA . $0000031378.29215 .0 \mathrm{C}$.

Guerrero, G., Smolarkiewicz, P. K., de Gouveia Dal Pino, E. M., Kosovichev, A. G., \& Mansour, N. N. (2016). On the role of tachoclines in solar and stellar dynamos. ApJ , 819, 1-17. doi 10.3847/0004-637X/819/2/104

Jones, C. A., Boronski, P., Brun, A. S., Glatzmaier, G. A., Gastine, T., Miesch, M. S., \& Wicht, J. (2011). Anelastic convection-driven dynamo benchmarks. Icarus, 216, 120-135. doi $10.1016 / \mathrm{j}$. icarus.2011.08.014.

Käpylä, P. J., Käpylä, M. J., \& Brandenburg, A. (2014). Confirmation of bistable stellar differential rotation profiles. A\&A, 570, 1-10. doi $10.1051 / 0004-6361 / 201423412$.

Käpylä, P. J., Mantere, M. J., \& Brandenburg, A. (2013). Oscillatory large-scale dynamos from Cartesian convection simulations. Geophys. Astro Fluid, 107, 244-257. doi:10.1080/03091929.2012.715158.

Käpylä, P. J., Mantere, M. J., Guerrero, G., Brandenburg, A., \& Chatterjee, P. (2011). Reynolds stress and heat flux in spherical shell convection. A\&A, 531, 1-17. doi:10.1051/0004-6361/201015884.

Karak, B. B., Käpylä, P. J., Käpylä, M. J., Brandenburg, A., Olspert, N., \& Pelt, J. (2015). Magnetically controlled stellar differential rotation near the transition from solar to anti-solar profiles. A\&A, 576, 1-17. doi $10.1051 / 0004-6361 / 201424521$. 
Kitchatinov, L. L., \& Ruediger, G. (1995). Differential rotation in solar-type stars: revisiting the Taylor-number puzzle. A\&A, 299, 446-452.

Lanza, A. F., Das Chagas, M. L., \& De Medeiros, J. R. (2014). Measuring stellar differential rotation with high-precision space-borne photometry. A\&A, 564, 1-15. doi http://dx.doi.org/10.1051/0004-6361/201323172.

Lawson, N., Strugarek, A., \& Charbonneau, P. (2015). Evidence of active MHD instability in EULAG-MHD simulations of solar convection. ApJ, 813, 1-13. doi: $10.1088 / 0004-637 X / 813 / 2 / 95$

Masada, Y., \& Sano, T. (2014). Long-term evolution of large-scale magnetic fields in rotating stratified convection. PASJ, 66, 1-7. doi $10.1093 / \mathrm{pasj} / \mathrm{psu081}$

Matt, S. P., Do Cao, O., Brown, B. P., \& Brun, A. S. (2011). Convection and differential rotation properties of $\mathrm{G}$ and $\mathrm{K}$ stars computed with the ASH code. $A N$, 332, 897-906. doi 10.1002/asna.201111624

McIntyre, M. E. (2007). Magnetic confinement and the sharp tachopause. In D. W. Hughes, R. Rosner, \& N. O. Weiss (Eds.), The Solar Tachocline, Cambridge University Press (p. 183).

Metcalfe, T. S., Monteiro, M. J. P. F. G., Thompson, M. J., Molenda-Żakowicz, J., Appourchaux, T., Chaplin, W. J., Doğan, G., Eggenberger, P., Bedding, T. R., Bruntt, H., Creevey, O. L., Quirion, P.-O., Stello, D., Bonanno, A., Silva Aguirre, V., Basu, S., Esch, L., Gai, N., Di Mauro, M. P., Kosovichev, A. G., Kitiashvili, I. N., Suárez, J. C., Moya, A., Piau, L., García, R. A., Marques, J. P., Frasca, A., Biazzo, K., Sousa, S. G., Dreizler, S., Bazot, M., Karoff, C., Frandsen, S., Wilson, P. A., Brown, T. M., Christensen-Dalsgaard, J., Gilliland, R. L., Kjeldsen, H., Campante, T. L., Fletcher, S. T., Handberg, R., Régulo, C., Salabert, D., Schou, J., Verner, G. A., Ballot, J., Broomhall, A.-M., Elsworth, Y., Hekker, S., Huber, D., Mathur, S., New, R., Roxburgh, I. W., Sato, K. H., White, T. R., Borucki, W. J., Koch, D. G., \& Jenkins, J. M. 
(2010). A precise asteroseismic age and radius for the evolved Sun-like star KIC 11026764. ApJ, 723, 1583-1598. doi 10.1088/0004-637X/723/2/1583

Miesch, M. S., Brun, A. S., \& Toomre, J. (2006). Solar Differential Rotation Influenced by Latitudinal Entropy Variations in the Tachocline. ApJ, 641, 618-625. doi:10.1086/499621

Miesch, M. S., Elliott, J. R., Toomre, J., Clune, T. L., Glatzmaier, G. A., \& Gilman, P. A. (2000). Three-dimensional Spherical Simulations of Solar Convection. I. Differential Rotation and Pattern Evolution Achieved with Laminar and Turbulent States. ApJ, 532, 593-615. doi:10.1086/308555.

Morel, P. (1997). CESAM: A code for stellar evolution calculations. Ap\&SS, 124, 597-614. doi:10.1051/aas: 1997209

J.-D. do Nascimento, J., Garca, R. A., Mathur, S., Anthony, F., Barnes, S. A., Meibom, S., da Costa, J. S., Castro, M., Salabert, D., \& Ceillier, T. (2014). Rotation periods and ages of solar analogs and solar twins revealed by the Kepler Mission. ApJ , 790, 1-5. URL: http://stacks .iop.org/2041-8205/790/i=2/a=L23 doi $10.1088 / 2041-8205 / 790 / 2 /$ L23

Nelson, N. J., Brown, B. P., Brun, A. S., Miesch, M. S., \& Toomre, J. (2013). Magnetic Wreaths and Cycles in Convective Dynamos. ApJ, 762, 1-20. doi $10.1088 / 0004-637 \mathrm{X} / 762 / 2 / 73$

Noyes, R. W., Hartmann, L. W., Baliunas, S. L., Duncan, D. K., \& Vaughan, A. H. (1984a). Rotation, convection, and magnetic activity in lower mainsequence stars. ApJ, 279, 763-777. doi $10.1086 / 161945$.

Noyes, R. W., Weiss, N. O., \& Vaughan, A. H. (1984b). The relation between stellar rotation rate and activity cycle periods. ApJ, 287, 769-773. doi $10.1086 / 162735$.

Oláh, K., Kolláth, Z., Granzer, T., Strassmeier, K. G., Lanza, A. F., Järvinen, S., Korhonen, H., Baliunas, S. L., Soon, W., Messina, S., \& Cutispoto, G. 
(2009). Multiple and changing cycles of active stars. A\&A, 501, 703-713. doi $10.1051 / 0004-6361 / 200811304$

Pallavicini, R., Golub, L., Rosner, R., Vaiana, G. S., Ayres, T., \& Linsky, J. L. (1981). Relations among stellar X-ray emission observed from Einstein, stellar rotation and bolometric luminosity. ApJ, 248, 279-290. doi:10.1086/159152.

Pizzolato, N., Maggio, A., Micela, G., Sciortino, S., \& Ventura, P. (2003). The stellar activity-rotation relationship revisited: Dependence of saturated and non-saturated X-ray emission regimes on stellar mass for late-type dwarfs. $A \& A, 397,147-157$. doi 10.1051/0004-6361:20021560.

Plachinda, S. I., \& Tarasova, T. N. (1999). Precise Spectropolarimetric Measurements of Magnetic Fields on Some Solar-like Stars. ApJ, 514, 402-410. doi $10.1086 / 306912$

Racine, E., Charbonneau, P., Ghizaru, M., Bouchat, A., \& Smolarkiewicz, P. K. (2011). On the mode of dynamo action in a global large-eddy simulation of solar convection. ApJ, 735, 1-22. URL: http://stacks.iop.org/0004-637X/735/i=1/a=46 doi $10.1088 / 0004-637 X / 735 / 1 / 46$

Reinhold, T., Reiners, A., \& Basri, G. (2013). Rotation and differential rotation of active Kepler stars. $A \& A, 560,1-19$. doi $10.1051 / 0004-6361 / 201321970$.

Saar, S. H. (1990). Spectroscopic measurements of magnetic fields on solar-like stars - Techniques and results. Mem. Soc. Astron. Ital., 61, 559-575.

Saar, S. H., \& Brandenburg, A. (1999). Time Evolution of the Magnetic Activity Cycle Period. II. Results for an Expanded Stellar Sample. ApJ, 524, 295-310. doi $10.1086 / 307794$.

Spiegel, E. A., \& Zahn, J.-P. (1992). The solar tachocline. A\&A, 265, 106-114.

Spruit, H. C. (2003). Origin of the torsional oscillation pattern of solar rotation. Sol. Phys., 213, 1-21. doi 10.1023/A:1023202605379. 
Strugarek, A., Brun, A. S., \& Zahn, J.-P. (2011). Magnetic confinement of the solar tachocline: II. Coupling to a convection zone. A\&A, 532, 1-15. doi:10.1051/0004-6361/201116518

Vasil, G. M., Lecoanet, D., Brown, B. P., Wood, T. S., \& Zweibel, E. G. (2013). Energy Conservation and Gravity Waves in Sound-proof Treatments of Stellar Interiors. II. Lagrangian Constrained Analysis. ApJ, 7773, 1-23. doi:10.1088/0004-637X/773/2/169, arXiv:1303.0005

Vida, K., Oláh, K., \& Szabó, R. (2014). Looking for activity cycles in latetype Kepler stars using timefrequency analysis. MNRAS, 441, 2744-2753. doi $10.1093 / \mathrm{mnras} / \mathrm{stu} 760$

Zahn, J.-P. (1992). Circulation and turbulence in rotating stars. A\&A, 265, $115-132$. 


\begin{tabular}{c|ccccccc} 
Model & $M 05_{d 1}$ & $M 07_{s}$ & $M 09_{s}$ & $M 09_{d 1}$ & $M 09_{d 3}$ & $M 11_{d 1}$ & $M 11_{d 3}$ \\
\hline$r_{\text {in }}\left(10^{10} \mathrm{~cm}\right)$ & 0.39 & 1.41 & 2.25 & 2.25 & 2.25 & 4.27 & 4.27 \\
$r_{\text {out }}\left(10^{10} \mathrm{~cm}\right)$ & 2.90 & 4.30 & 5.72 & 5.72 & 5.72 & 8.34 & 8.34 \\
$D\left(10^{10} \mathrm{~cm}\right)$ & 2.51 & 2.89 & 3.47 & 3.47 & 3.47 & 4.07 & 4.07 \\
$r_{b c r} / r_{\text {out }}$ & 0.58 & 0.68 & 0.71 & 0.71 & 0.71 & 0.76 & 0.76 \\
$\nu\left(10^{12} \mathrm{~cm}^{2} / \mathrm{s}\right)$ & 0.45 & 5.31 & 14.3 & 10.01 & 5.81 & 38.01 & 20.95 \\
$\kappa\left(10^{13} \mathrm{~cm}^{2} / \mathrm{s}\right)$ & 0.18 & 2.12 & 5.72 & 4.02 & 2.32 & 15.20 & 8.78 \\
$\eta\left(10^{12} \mathrm{~cm}^{2} / \mathrm{s}\right)$ & 0.22 & 5.31 & 14.30 & 5.03 & 5.81 & 38.01 & 21.95 \\
$\Omega / \Omega_{\odot}$ & 1 & 0.3 & 0.5 & 1 & 3 & 1 & 3 \\
$T a\left(10^{4}\right)$ & 134.4 & 0.15 & 0.12 & 0.99 & 2.65 & 0.13 & 3.51 \\
$R a\left(10^{6}\right)$ & 19.27 & 0.76 & 0.09 & 0.21 & 1.03 & 0.02 & 0.05 \\
$P r$ & 0.25 & 0.25 & 0.25 & 0.25 & 0.25 & 0.25 & 0.25 \\
$P r_{m}$ & 2 & 1 & 1 & 2 & 1 & 1 & 1 \\
$\tau_{\eta}($ years $)$ & 9.21 & 0.51 & 0.27 & 0.77 & 0.67 & 0.14 & 0.24 \\
$\tau_{c}($ years $)$ & 1.01 & 0.45 & 0.30 & 0.30 & 0.30 & 0.16 & 0.16 \\
$\tilde{v_{r}}(\mathrm{~m} / \mathrm{s})$ & 8 & 20 & 35 & 35 & 35 & 80 & 80 \\
$\tilde{v}(\mathrm{~m} / \mathrm{s})$ & 66 & 55 & 110 & 154 & 233 & 305 & 484 \\
$\tilde{B}\left(10^{3} \mathrm{G}\right)$ & 1.1 & 0.8 & 2.3 & 4.2 & 7.2 & 4.5 & 9.5 \\
$R o$ & 0.31 & 1.84 & 1.68 & 0.84 & 0.28 & 1.17 & 0.39 \\
$R e$ & 370 & 30 & 27 & 53 & 139 & 33 & 90 \\
$R e_{m}$ & 740 & 30 & 27 & 106 & 139 & 33 & 90 \\
$\Lambda\left(10^{-3}\right)$ & 0.03 & 0.16 & 1.29 & 1.55 & 1.00 & 10.74 & 10.05
\end{tabular}

Table 1: Models parameters. $r_{i n}$ is the inner radius, $r_{\text {out }}$ the outer radius, $r_{b c z}$ the base of the convective zone and $D=r_{\text {out }}-r_{\text {in }}$ the radial length of the simulation domain. $\nu, \kappa$ and $\eta$ are the effective eddy diffusivities of the momentum, heat and magnetic field transport. $\Omega$ is the rotation rate. $T a=4 \Omega^{2} D^{4} / \nu^{2}$ is the Taylor number. $R a=(-\partial \bar{\rho} / \partial S) \Delta S g D^{3} / \rho \nu \kappa$ is the Rayleigh number with $S$ the entropy and $\rho$ the density ( $\bar{\rho}$ means the background state of the density). $\operatorname{Pr}=\nu / \kappa$ is the Prandtl number. $P r_{m}=\nu / \eta$ is the magnetic Prandtl number. $R o=\tilde{v} / 2 \Omega D$ is the Rossby number with $\tilde{v}$ the rms velocity in the covection zone. $R e=\tilde{v} D / \nu$ is the Reynolds number with $\tilde{v}$ the rms velocity in the convection zone. $\operatorname{Re}_{m}=\tilde{v} D / \eta$ is the magnethe Reynolds number. $\Lambda=\tilde{B}^{2} / 8 \pi \bar{\rho} D \tilde{v} \Omega$ is the Elsasser number with $\tilde{B}$ the rms magnetic field strength in the convection zone. $\tau_{\eta}=D^{2} / \pi^{2} \eta$ is the ohmic diffusion time. $\tau_{c}=D / \tilde{v_{r}}$ is the overturning convection time with $\tilde{v}_{r}$ the radial component of the averaged velocity in the convection zone. The models resolution is $\left(N_{r}, N_{\theta}, N_{\phi}\right) 769 \times 256 \times 512$ except the 


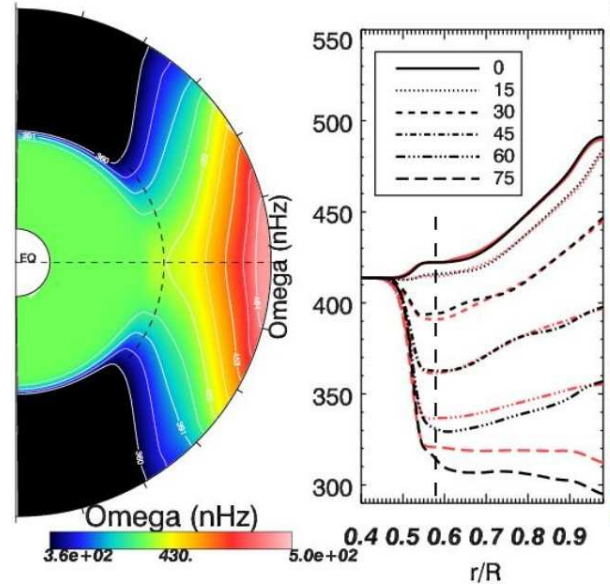

(a) $M 05_{d 1}$

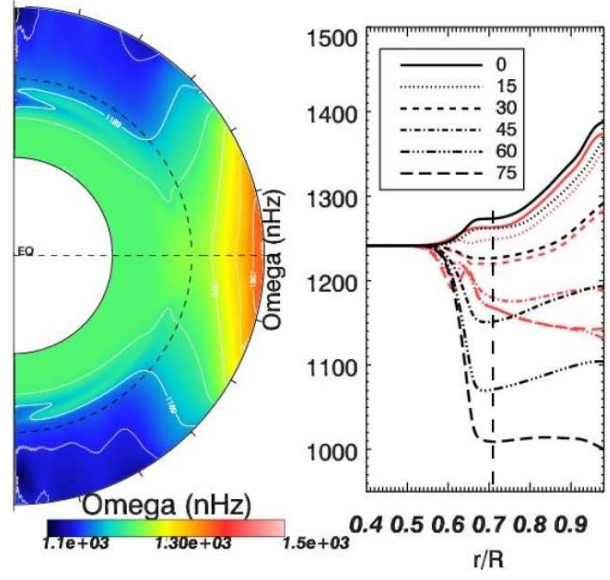

(c) $M 09_{d 3}$
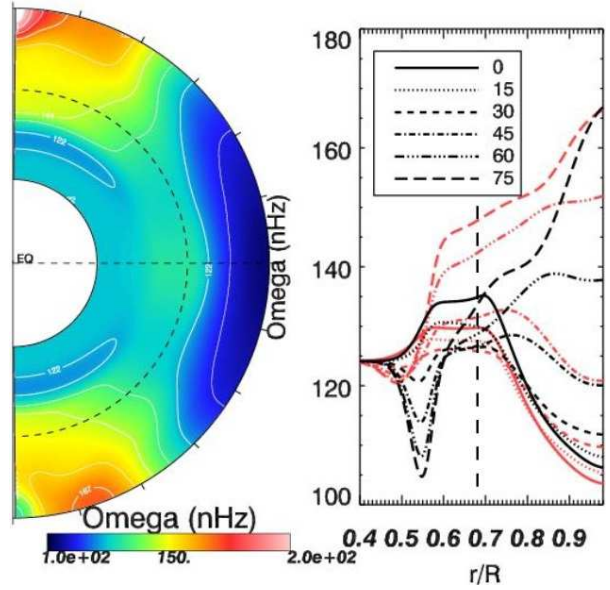

(e) $M 07_{s}$

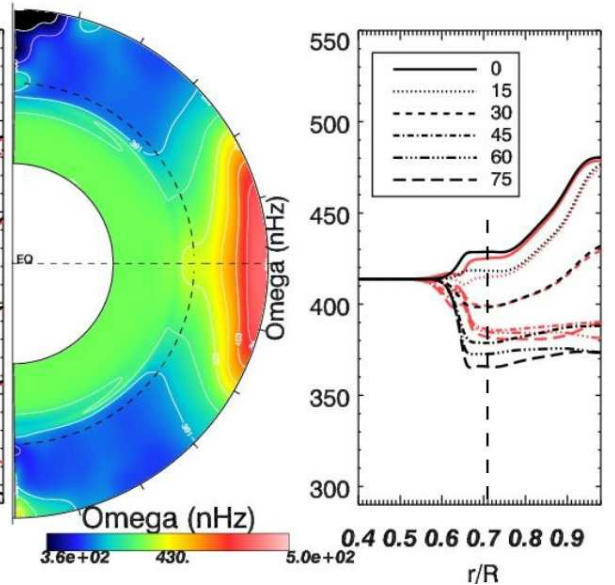

(b) $M 09_{d 1}$

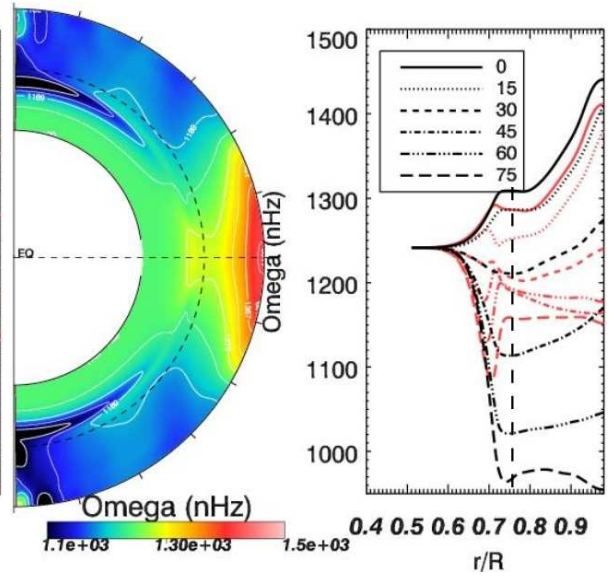

(d) $M 11_{d 3}$

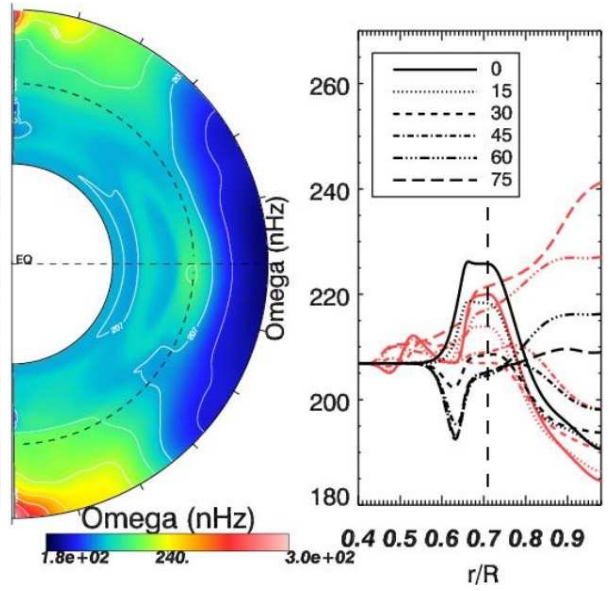

(f) $M 09_{s}$

31

Figure 2: Temporal and longitudinal averaged of the angular velocity profiles during $10 \tau_{c}$ and radial cuts from the equator to the latitude $75^{\circ}$ each $15^{\circ}$ (black lines are the hydro cases and the red lines the MHD cases) between 0.4 and 0.96 $r / r_{*}\left(r / r_{*}=0.4\right.$ is not necessarily $\left.r=r_{b c r}\right)$. The long dashed vertical line on the right hand side panel of each cases shown the basis of the convective layer. 

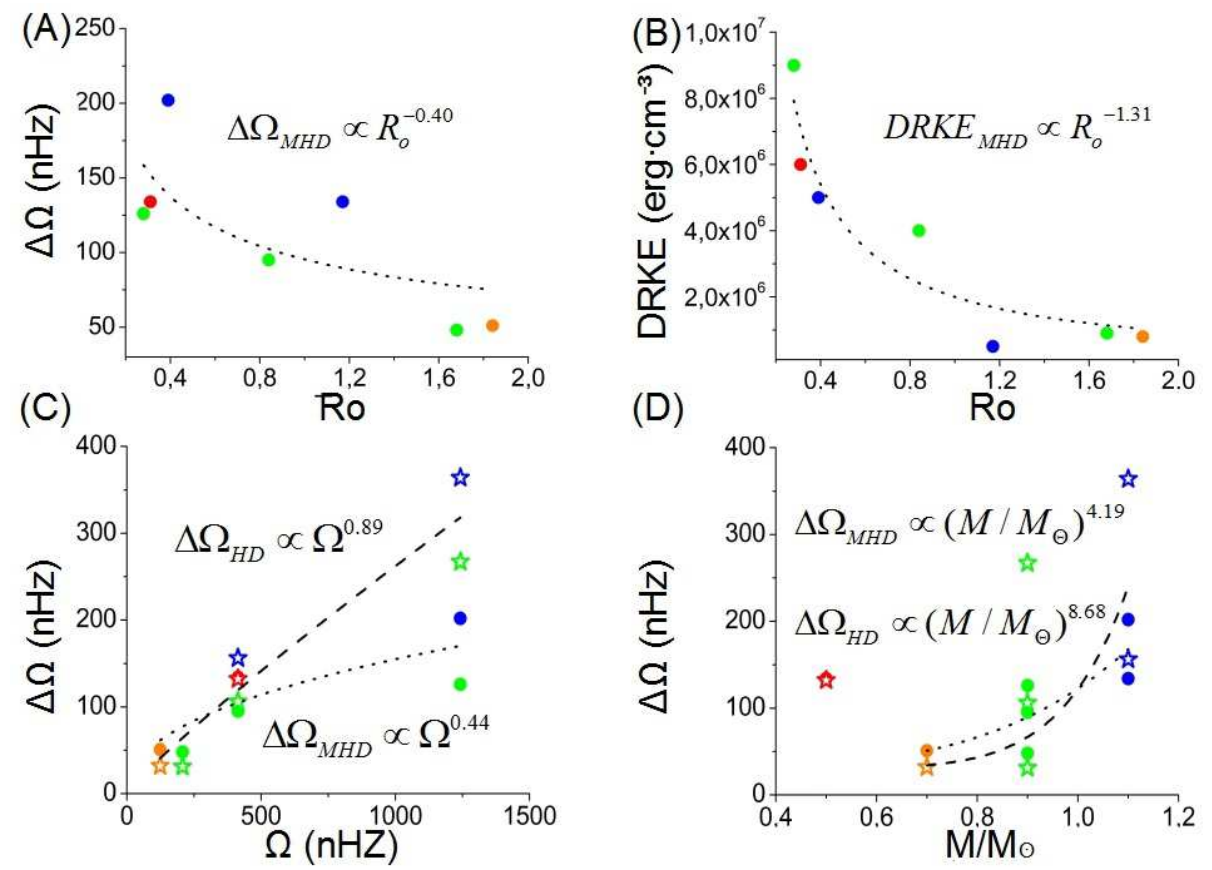

\begin{tabular}{|ll|}
\hline MHD trend $\cdots$ & Hydro trend $-\cdots$ \\
$0.5 \mathrm{M} \odot$ & $\star 0.5 \mathrm{M} \odot$ \\
$0.7 \mathrm{M}$ & $\star 0.7 \mathrm{M} \odot$ \\
$0.9 \mathrm{M}$ & $\star 0.9 \mathrm{M} \odot$ \\
$01.1 \mathrm{M} \odot$ & $\star 1.1 \mathrm{M} \odot$ \\
\hline
\end{tabular}

Figure 3: Absolute value of the differential rotation between the equator and $60^{\circ}$ latitude (A) and differential rotation kinetic energy versus Ro (B). Differential rotation versus rotation (C). Differential rotation versus mass (D). MHD data are the solid circles and hydro data are the empty circles. The dotted (dashed) line shows the linear fit of the MHD (Hydro) data. The data sets of the graphs are fitted to power equations $A=a+\alpha B^{\beta}$ ( $\alpha, \beta$ and $a$ parameters are fitted). 


\begin{tabular}{c|ccccccc} 
Model & $M 05_{d 1}$ & $M 07_{s}$ & $M 09_{s}$ & $M 09_{d 1}$ & $M 09_{d 3}$ & $M 11_{d 1}$ & $M 11_{d 3}$ \\
\hline $\operatorname{KE~}\left(10^{6}\right)$ & $49.8(>99 \%)$ & $5.1(>99 \%)$ & $4.8(99 \%)$ & $6.3(97 \%)$ & $10.0(95 \%)$ & $2.4(71 \%)$ & $7.1(92 \%)$ \\
$\operatorname{DRKE}\left(10^{6}\right)$ & $42.6(86 \%)$ & $1.0(20 \%)$ & $0.7(15 \%)$ & $3.1(49 \%)$ & $8.9(89 \%)$ & $0.7(29 \%)$ & $5.8(82 \%)$ \\
$\operatorname{CKE}\left(10^{6}\right)$ & $5.4(11 \%)$ & $4.0(78 \%)$ & $3.5(73 \%)$ & $3.1(50 \%)$ & $1.0(10 \%)$ & $1.7(59 \%)$ & $1.2(17 \%)$ \\
$\operatorname{MCKE}\left(10^{6}\right)$ & $1.8(3 \%)$ & $0.1(2 \%)$ & $0.6(12 \%)$ & $0.1(1 \%)$ & $0.1(1 \%)$ & $0.0(>1 \%)$ & $0.1(1 \%)$ \\
$\operatorname{ME}\left(10^{5}\right)$ & $0.14(<1 \%)$ & $0.45(<1 \%)$ & $0.39(1 \%)$ & $2.13(3 \%)$ & $5.11(5 \%)$ & $3.77(29 \%)$ & $6.02(8 \%)$ \\
$\operatorname{PME~}\left(10^{5}\right)$ & $0.00(<1 \%)$ & $0.01(2 \%)$ & $0.09(23 \%)$ & $0.02(1 \%)$ & $0.09(2 \%)$ & $0.28(7 \%)$ & $0.12(2 \%)$ \\
$\operatorname{TME}\left(10^{5}\right)$ & $0.11(79 \%)$ & $0.35(78 \%)$ & $0.22(56 \%)$ & $1.92(90 \%)$ & $4.06(79 \%)$ & $2.23(60 \%)$ & $5.03(84 \%)$ \\
$\operatorname{FME~}\left(10^{5}\right)$ & $0.03(21 \%)$ & $0.09(20 \%)$ & $0.08(21 \%)$ & $0.19(9 \%)$ & $0.96(19 \%)$ & $1.26(33 \%)$ & $0.83(14 \%)$ \\
$\Delta \Omega(\mathrm{nHz}) \mathrm{MHD}$ & 134 & -51 & -48 & 95 & 126 & -134 & 202 \\
$\Delta \Omega(\mathrm{nHz}) \mathrm{HD}$ & 132 & -32 & -31 & 106 & 267 & -156 & 364 \\
$\Delta \Omega / \Omega(\%)(\mathrm{MHD})$ & 32 & 41 & 23 & 23 & 10 & 32 & 16 \\
$\Delta \Omega / \Omega(\%)(\mathrm{HD})$ & 32 & 26 & 15 & 26 & 21 & 38 & 29
\end{tabular}

Table 2: Time averaged of the kinetic energy (KE) divided in axisymmetric differential rotation (DRKE), non axisymmetric convective (CKE) and axisymmetric meridional circulation (MCKE) components. The magnetic energy (ME) is divided in toroidal (TME) and poloidal (PME) components. Energy units are ergs $\mathrm{cm}^{-3}$. Time averaged differential rotation and normalized values by the star's rotation $\Delta \Omega / \Omega$ for the hydro and MHD simulations. 

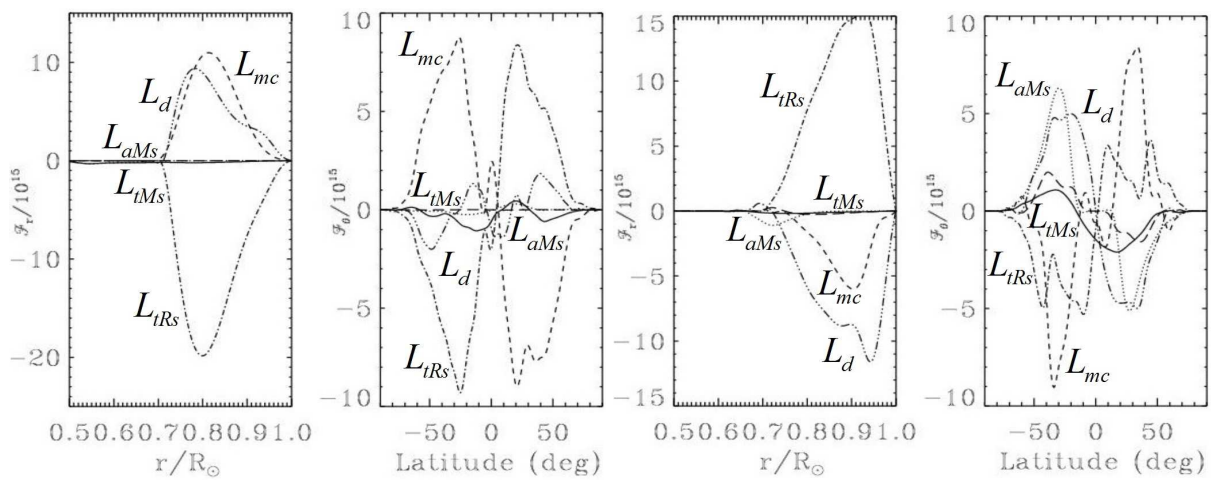

(a) $M 09_{s}$ (anti-solar)

(b) $M 09_{d 3}$ (solar)

Figure 4: Time average of the latitudinal line integral of the angular momentum flux $\mathcal{F}_{r}(r)$ (on the left) and radial line integral of the angular momentum flux $\mathcal{F}_{\theta}(\theta)$ (on the right). Model $M 09_{s}$ (top graphs) and $M 09_{d 3}$ (bottom graphs). The fluxes are decomposed in different contributions: viscous diffusion $\left(L_{d}\right.$ dashdot-dot-dot line), turbulent Reynolds stresses ( $L_{t R s}$, dash-dot line), meridional circulation $\left(L_{m c}\right.$, dashed line), axisymmetric $\left(L_{a M s}\right.$, dotted line) and turbulent ( $L_{t M s}$, long dashed line) Maxwell stresses. The solid line shows the total fluxes. The flux is averaged over $10 \tau_{c}$. 


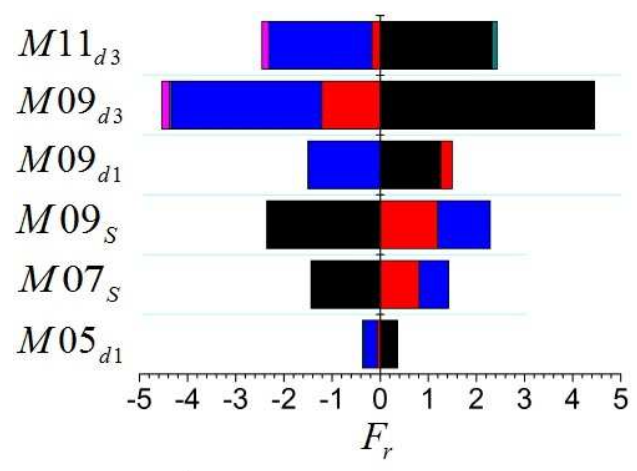

- Viscous diffusion

- Meridional circulation

- Non axysimmetric Reynolds Stresses

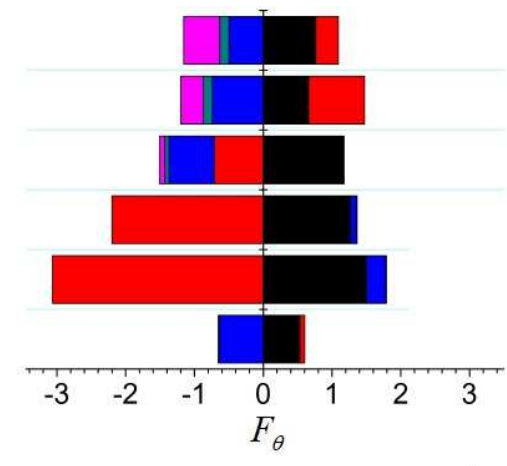

- Magnetic axysimmetric Reynolds Stresses

- Magnetic non axysimmetric Reynolds Stresses

Figure 5: Radial (A) and latitudinal (B) net angular momentum fluxes integrated in radius and latitude respectively (normalized to $10^{18}$ ). The subindex "S" indicates the anti-solar models.

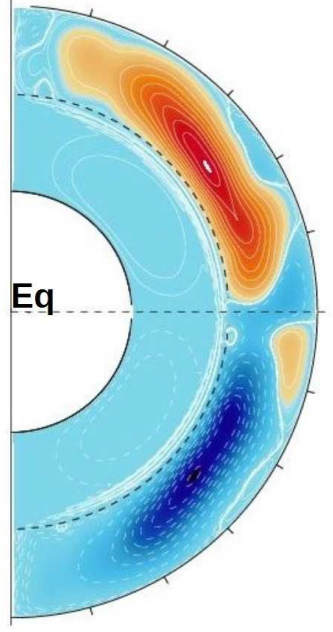

(a) $M 09_{s}$ (anti-solar)

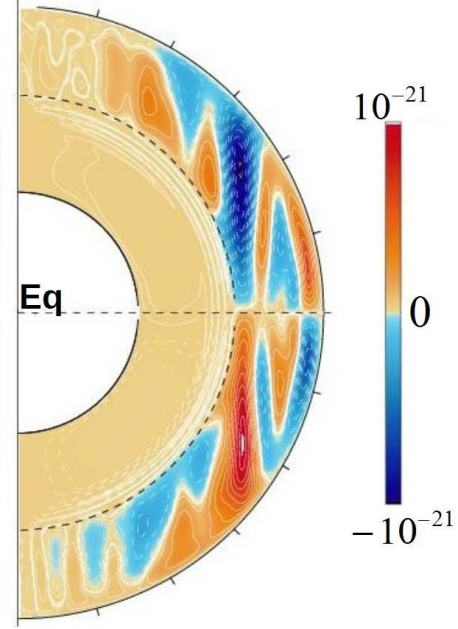

(b) $M 09_{d 3}$ (solar)

Figure 6: Temporal and longitudinal averaged meridional circulation and contours of the stream function $\psi$ for the models $M 09_{s}$ and $M 09_{d 3}$ during $10 \tau_{c}$. Units in $\mathrm{g} / \mathrm{s}$. 

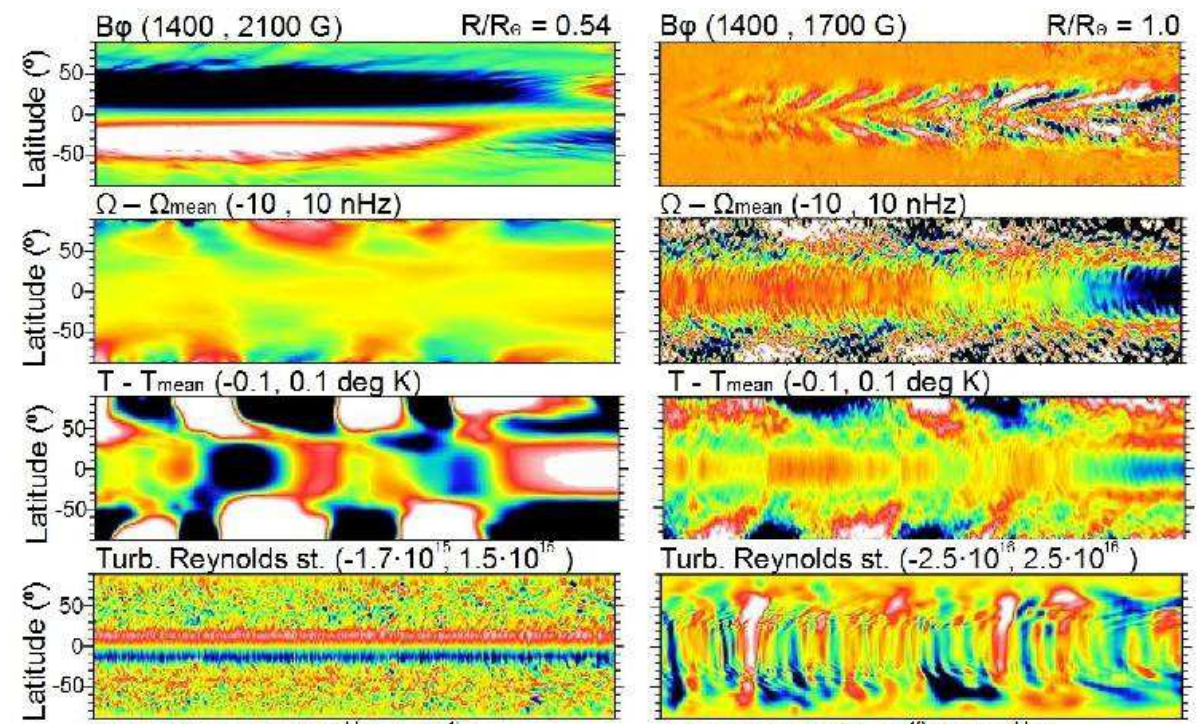

$T-T_{\text {mean }}(-0.1,0.1 \mathrm{deg} K)$

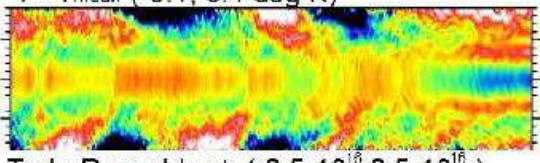

Turb. Reynolds st. $\left(-2.5 \cdot 10^{16}, 2.5 \cdot 10^{16}\right)$
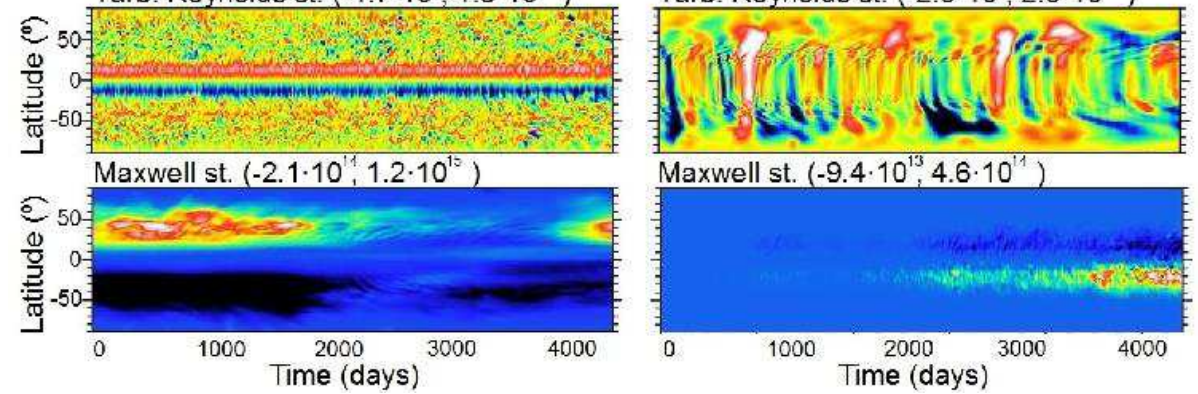

Figure 7: Model $M 05_{d 1}$. Time-latitude diagrams of the $B_{\varphi}$ component of the magnetic field, the torsional oscillations, temperature oscillations as well as the turbulent Reynolds stresses and the Maxwell stresses components of the latitudinal angular momentum flux (dyn.cm) at the tachocline $\left(r / r_{*}=0.54\right.$, left panels) and the top of the convective layer ( $r=r_{\text {out }}$, right panels) 

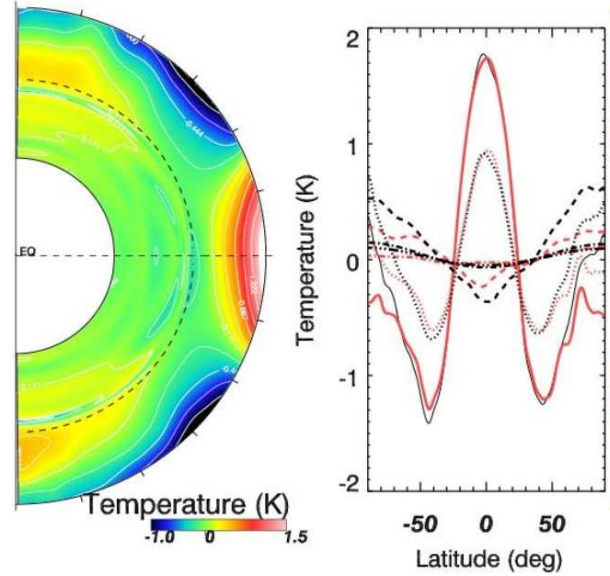

(a) Temperature $M 09_{s}$ model
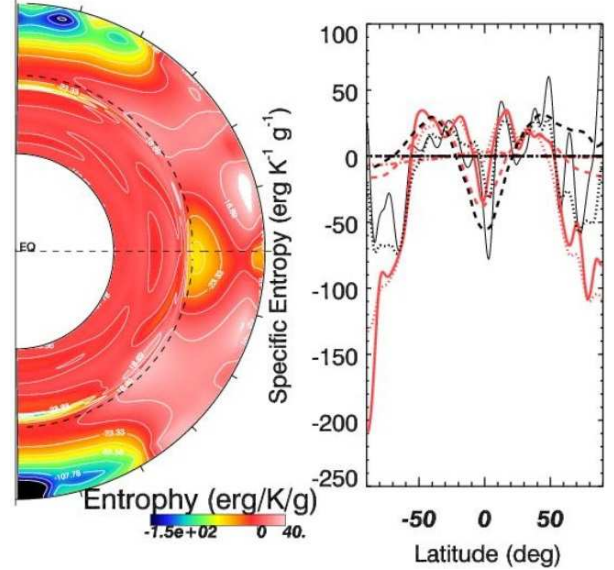

(c) Entropy $M 09_{s}$ model

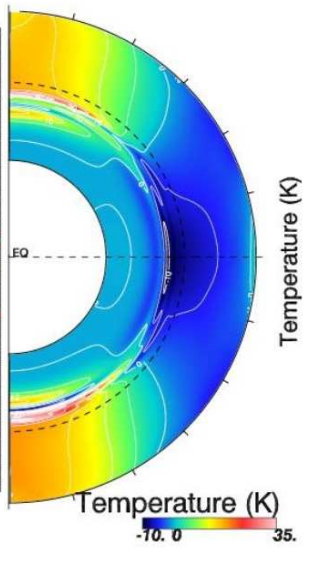

(b) Temperature $M 09_{d 3}$ model

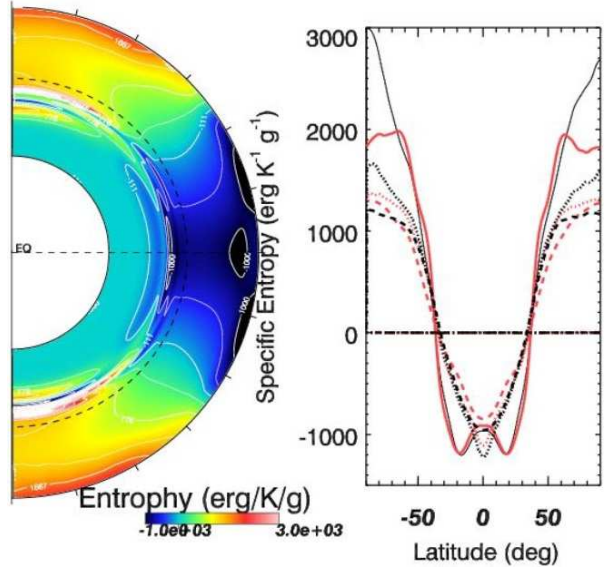

(d) Entropy $M 09_{d 3}$ model

Figure 8: Temporal and longitudinal averaged of temperature and entropy profiles during $10 \tau_{c}$, including latitudinal cuts at radius $r_{\text {out }}$ (solid line), $3 \cdot r_{\text {out }} / 4$ (dotted line), $r_{\text {out }} / 2$ (dashed line), $r_{\text {out }} / 4$ (dash-dot line), and $r_{\text {in }}$ (dash-dotdot-dot line) (black lines are the hydro cases and the red lines the MHD cases). 


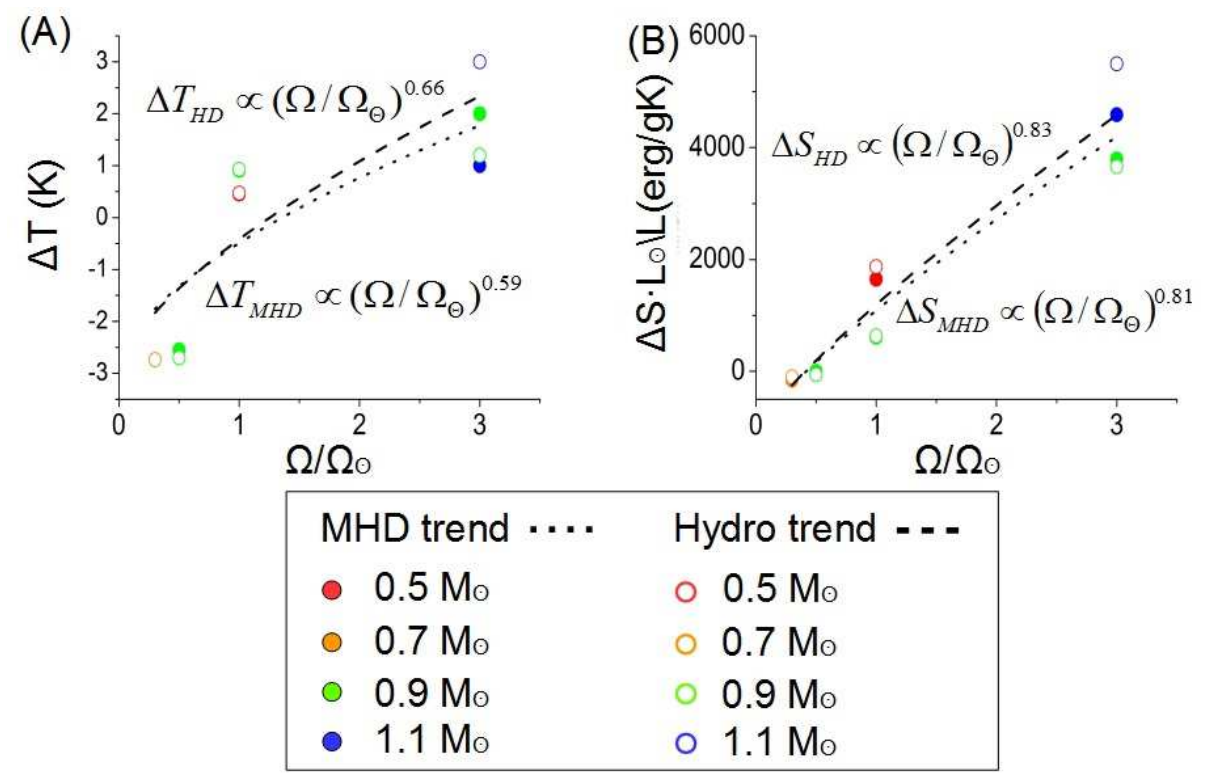

Figure 9: Trends of the temperature (A) and entropy contrasts respect to the star's rotation at the top of the convective layer. Entropy contrast is weighted by the star's luminosity. The data sets of the graphs are fitted to power equations $A=c t t e+\alpha B^{\beta}$ ( $\alpha, \beta$ and ctte parameters are fitted) 


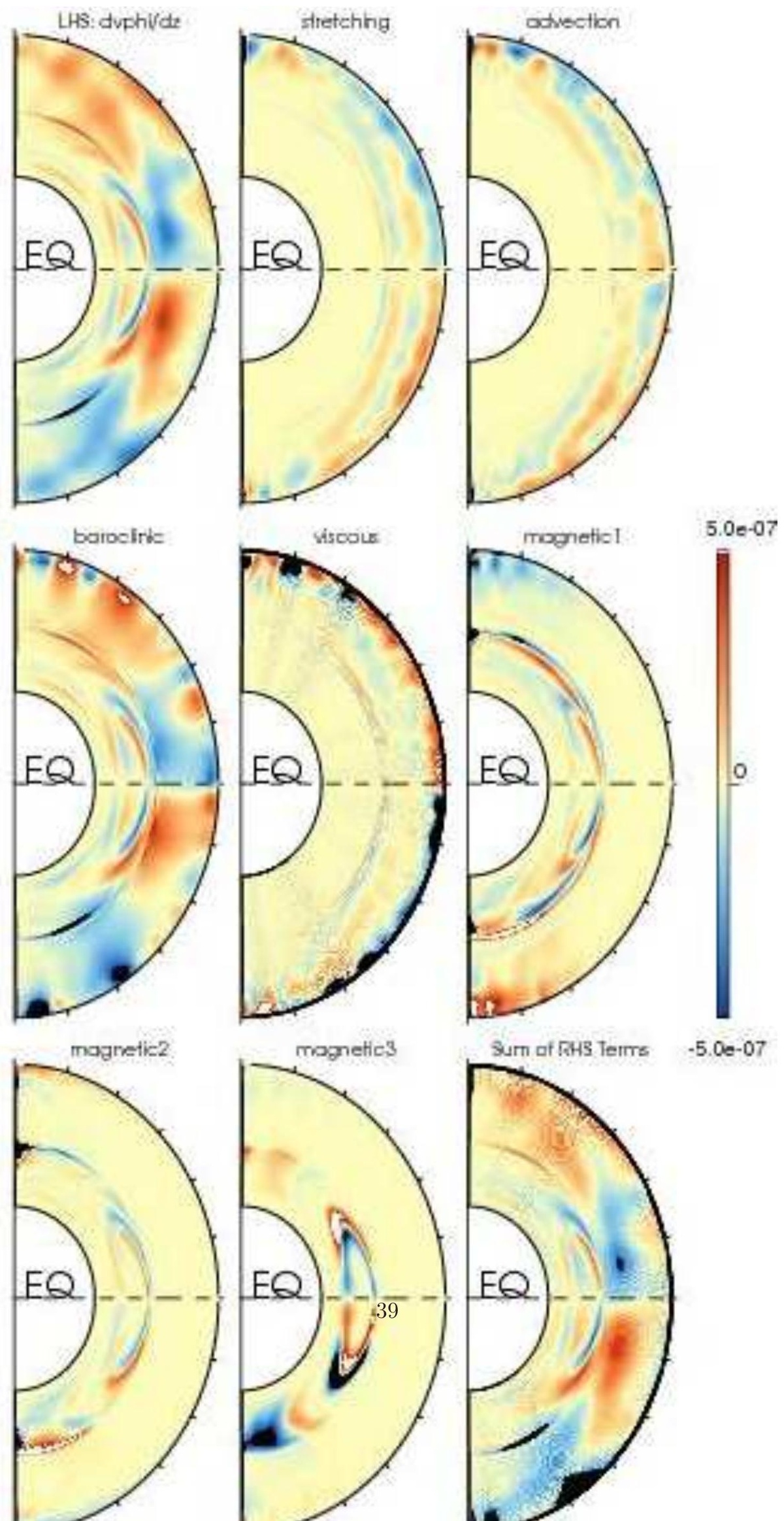



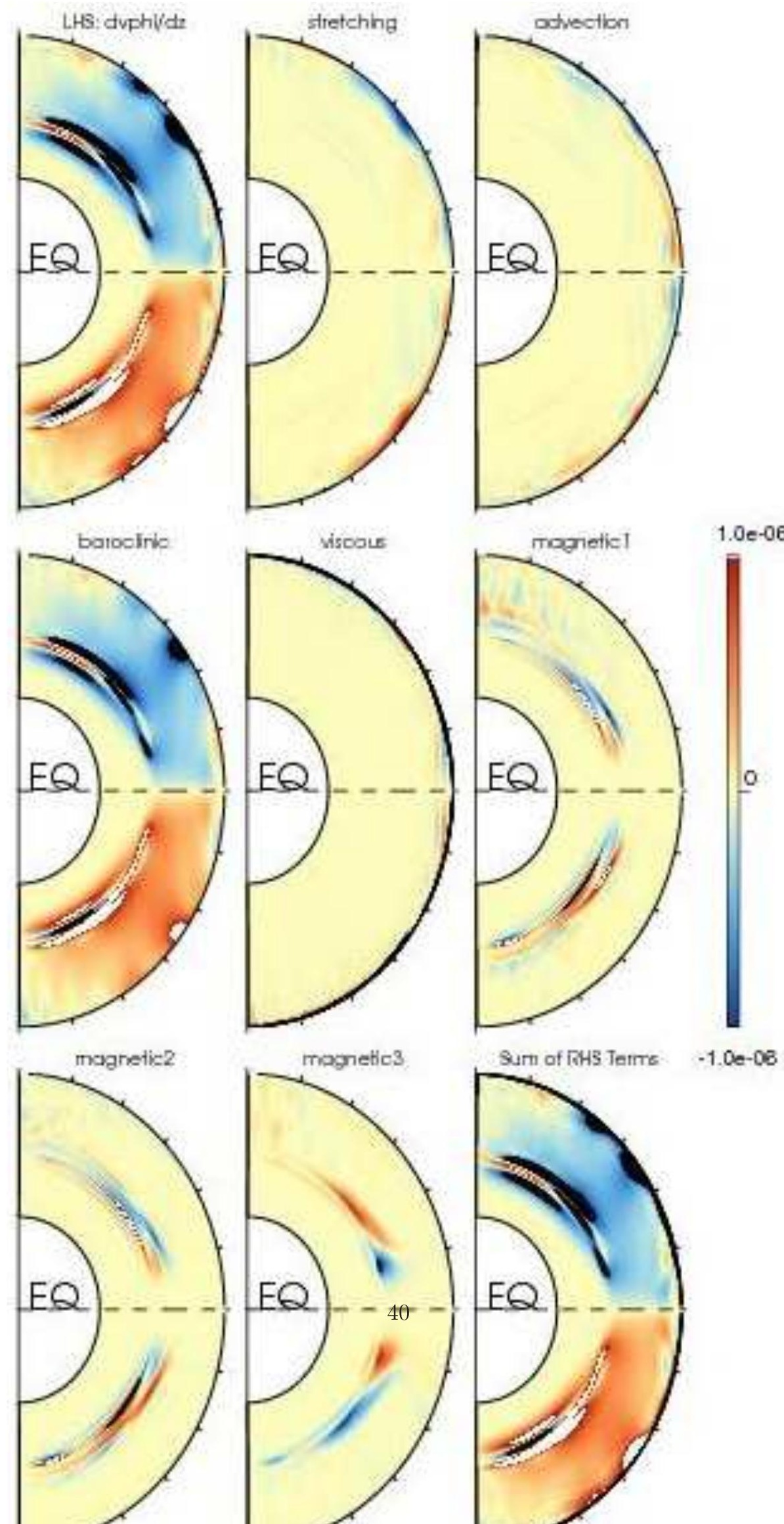